\title{
Effects of element orientation on apparent motion perception
}

\author{
P. WERKHOVEN, H. P. SNIPPE, and J. J. KOENDERINK \\ Utrecht University, Utrecht, The Netherlands
}

\begin{abstract}
We present an ambiguous motion paradigm that allows us to quantify the influence of aspects of form relevant to the perception of apparent motion. We report on the role of bar element orientation in motion paths. The effect of orientation differences between bar elements in a motion path is small with respect to the crucial role of the orientation of bar elements relative to motion direction. Motion perception between elements oriented along the motion direction dominates motion perception between elements oriented perpendicularly to motion direction. The perception of apparent motion is affected by bar length and width and is anisotropic.
\end{abstract}

Given a suitable choice of spatial and temporal parameters, apparent motion (spatiotemporal sampled motion) is perceptually equivalent to continuous motion and thus reveals some spatiotemporal characteristics of human visual motion detection (Burr, Ross, \& Morrone, 1986). This phenomenon is consistent with several computational and physiological models that exploit local spatial and temporal derivatives of luminance at a low resolution level (Koenderink \& van Doom, 1987; Marr, 1982). However, the stroboscopic nature of apparent motion makes it tempting to model it in terms of a bilocal spatiotemporal correlation process (Sperling, van Santen, \& Burt, 1985; van Santen \& Sperling, 1985).

Reichardt (1969), for example, has proposed a possible neural correlation mechanism that matches features of spatial structure (aspects of form) at different positions and moments in time. Some parameters of this model (such as optimal spatial and temporal intervals between presentations of image elements) have been studied for at least a century, and they have been psychophysically quantified in many experiments with human subjects (Braddick, 1974; Burt \& Sperling, 1981; Korte, 1915; Ramachandran \& Inada, 1985; Wertheimer, 1912). Less attention has been paid, however, to the aspects of the form of the image elements (geometrical relations, topological properties) that are basic to the correlation process.

In the vein of Bayes decision theory (Duda \& Hart, 1973 ), the motion detection problem can be posed in probabilistic terms. A tradeoff exists between the sensitivity of a motion detector to specific image element features (form, spatial frequency) and the performance in noisy situations, constrained by the a priori probability structure of the dynamic scenery. Specificity only to sim-

Correspondence should be addressed to Peter Werkhoven, Department of Medical and Physiological Physics, Buys Ballot Laboratory, Utrecht University, P.O. Box 80000, NL-3508 TA, The Netherlands. ple element features (like flux) maximizes the probability of detection even in situations in which the image is corrupted by noise or element features are changing during motion (e.g., 2-D transforming projections of 3-D motion). However, it also introduces false alarms (the matching of nonsimilar elements). The probability of error can be minimized by a high feature selectivity, so that motion detection will not suffer from false alarms, but misses (failures to detect motion) will occur if image elements undergo minor structural changes.

At the core of the correspondence problem lies the question of what independent features should be seen by the motion detection system, so that overall risk (with respect to human performance) is minimized in natural situations. Gorea and Papathomas (1988) proposed motion processing units, which integrate spatiotemporal information from subunits that display similar filtering properties in a multidimensional perceptual space. Certain features in this perceptual space are not "sensed" by these subunits. A motion detector thus projects this perceptual space to a lower dimensional space (the kernel of the projection). One would like to know the dimensionality of this kernel and the filtering properties that define it. In trying to answer this intriguing question, we already have recourse to a substantial but often contradictory literature.

At the beginning of this century, the role of image element features was scarcely studied. For a long time, the Gestaltists (Wertheimer, 1912) banned the application of the currently popular metaphor of feature analysis to apparent motion perception, due to its character as a kind of mental chemistry. The quality of "wholes" and the temporal characteristics in particular attracted the curiosity of these early scientists. A few decades later, however, Fernberger (1934) and Orlansky (1940) became the first to report effects of similarity (bar width) between bar segments on the perception of apparent motion. In 1971, Kolers and Pomerantz began a serious attempt to survey stimulus features that were relevant to the percep- 
tion of apparent motion. Using alternation between two stimulus positions, long interstimulus intervals, and, primarily, complex structured figures, they found no influence of figural aspects on the strength of apparent motion. Navon (1976), who substantially elaborated upon Kolers' experiments, found that "figural identity does not have any effect on determining the type of motion experienced, except when the figural analysis involved is relatively simple"' (p. 130). He expressed the opinion that when the load on the "figural recognition process" is small, figural analysis can keep up with motion analysis and is likely to affect the perception of motion. But it is not evident that figural complexity should be related to processing time. Recent evidence indeed reveals the relevance to apparent motion of luminance (Pantle \& Picciano, 1976), flux (Shechter, Hochstein, \& Hillman, 1988, 1989), spatial frequency (Green, 1986; Watson, 1986 ), proximity (Burt \& Sperling, 1981; Williams \& Sekuler, 1984), binocular disparity (Gorea \& Papathomas, 1988; Green \& Odom, 1986), color (Gorea \& Papathomas, 1988), and even topological invariants (Chen, 1985).

A feature of special interest is orientation. The experiments (Burt \& Sperling, 1981; Frisby, 1972; Gorea \& Papathomas, 1988; Green, 1986; Ullman, 1978) on the importance of orientation as a feature relevant to the correspondence process are very contradictory. Using alternating Gabor elements, Green (1986) showed that low spatial frequencies dominate the correspondence process and that the orientation of the elements is an important feature. Ullman (1980) also reported a strong effect of orientation difference of bar elements and related this to 3-D interpretations. On the other hand, Burt and Sperling (1981) concluded from their experiments on optimal spatiotemporal parameters that motion strength along a path with successive elements which differ in orientation or size is not necessarily weaker than motion along a path configured entirely of identical elements. Moreover, Gorea and Papathomas (1988) found that orientation differences cannot veto the percept of motion, while color and luminance can.

None of the aforementioned authors, however, has distinguished orientation differences between image elements from the orientation of image elements relative to motion direction, both of which were clearly intermingled in their experiments. The results of these experiments have traditionally been interpreted in terms of the quality of the match, depending on the orientation difference between two matched elements. The best match occurs between identical elements. Another possibility is to consider the orientation of each element relative to motion direction (not the orientation difference between elements) as the important feature affecting apparent motion. This leads to the suggestion that motion between differently oriented elements could be stronger than that between identical elements.

In this paper, we present the results of apparent motion experiments with oriented elements, in support of our claim that orientation relative to the motion direction plays an important role in the perception of apparent motion.

\section{EXPERIMENTS}

\section{Method}

Use was made of a multiframe, multielement apparent motion paradigm that was ambiguous insofar as matches between image elements were possible in orthogonal directions.

Each stimulus consisted of a series of frames $\left(f_{0}, f_{1}, \ldots\right)$ shown successively ( $42 \mathrm{msec}$ each). The first frame $\left(f_{0}\right)$ was configured of parallel diagonal rows of alternated Image Elements $A$ and $B$ (symbolically) at regular positions (see Figure 1).

In one diagonal row, the horizontally and vertically projected distances between the centers of two neighboring image elements were, respectively, $d_{h}$ and $d_{v}$. Frame $f_{n}$ was identical to frame $f_{0}$, except for a vertical translation over distance $n d_{v}$. The diagonal rows were positioned so that $f_{n}=f_{n+4}$ (only four frames were needed, independent of the motion duration and display time per frame).

When frames $f_{n}$ and $f_{n+1}$ were displayed in sequence, two possible matches between elements of frames $f_{n}$ and $f_{n+1}$ were possible. The first match was a vertical one between identical elements over distance $d_{\nu}$. The second match was a horizontal one between nonidentical elements over distance $d_{h}$ (other matches were only possible between elements at larger distances). Thus, two motion paths existed, a vertical homogeneous path between identical elements and a horizontal heterogeneous path between nonidentical elements.

A periodic diagonal row pattern (Figure 1) was used for various reasons. First, the pattern created with several rows approached the appearance of real textured surfaces as much as possible. Second, the use of periodic rows made it possible to produce a continuous animation by cyclically displaying a finite series of frames (for computer memory efficiency). Finally, the global form of one diagonal row of elements in isolation induced motion that was perpendicular to the diagonal row orientation, which could dominate the horizontal and vertical motion paths between image elements. The periodic row pattern, on the other hand, did not induce such a motion.

We expected that the probability of motion perception in either horizontal or vertical direction would depend on $d_{h}, d_{v}$ and on the similarity of the nonidentical image elements.

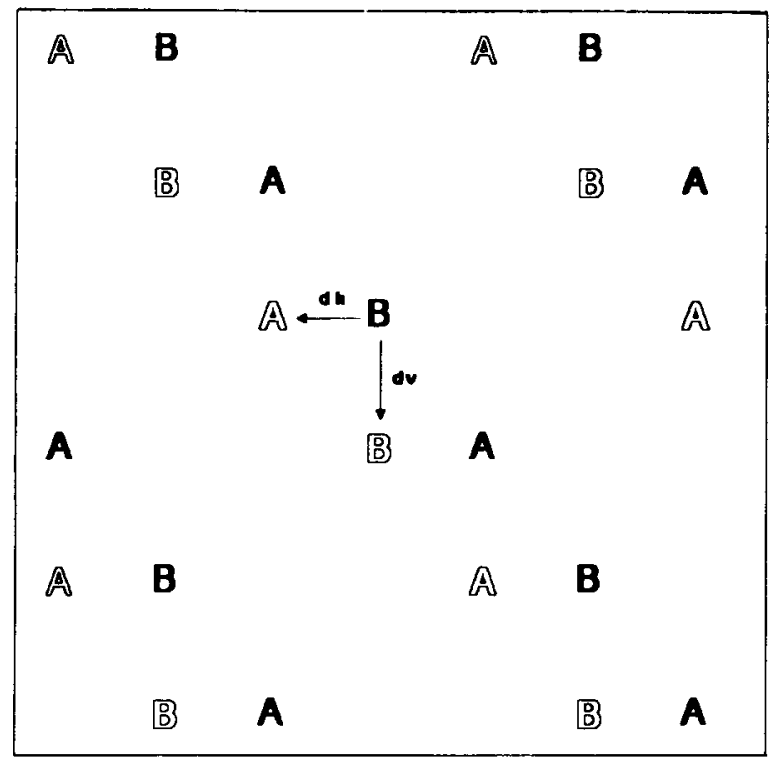

Figure 1. Schematic representation of elements (boldface characters) in one frame $\left(f_{n}\right)$ of an ambiguous motion display shifted downward over distance $d_{v}$, forming the elements (outlined characters) in the next frame $\left(f_{n+1}\right)$. 
The probability of perceiving motion between two elements is a monotonic decreasing function of the center-to-center distance between the two elements (Burt \& Sperling, 1981; Korte, 1915; Ullman, 1980). Similarity is operationally defined, an unknown function of physical differences between elements.

In our experiments, we measured the probability for horizontal motion perception as a function of the distance ratio $d_{\nu} / d_{h}$. This probability function increases monotonically as the distance ratio increases. We defined the $\boldsymbol{R}_{p s e}$ (the ratio at the point of subjective equality) as the distance ratio $d_{v} / d_{h}$ at which the probability for horizontal motion perception was $\mathbf{5 0 \%}$. The ratio $\boldsymbol{R}_{p s e}$ is a measure of the similarity of nonidentical elements with respect to the apparent motion processing system.

The ratio $R_{p s e}$ was determined in a session in which 100 stimuli were presented with varying $d_{v} / d_{h}$ ratios. The task of the subject was to report motion direction by pushing one of two buttons (horizontal/vertical). An elaborated "adaptive probit estimation" (Watt \& Andrews, 1981) procedure was used to estimate two parameters ( $\boldsymbol{R}_{p s e}$ and the slope at $\boldsymbol{R}_{p s e}$ ) of the psychometric function that would describe the performance of the observer.

It should be noted here that with this method an observer could reproduce $R_{p s e}$ values easily within a maximum range of $2 \%$.

The ratio of $d_{v}$ and $d_{h}$ can be assessed in several ways. We used two strategies, in different experiments. In the first, second, and fourth experiments, the sum of $d_{v}$ and $d_{h}$ was kept constant $\left(d_{\nu}+d_{h}=42.1^{\prime}\right)$ and the ratio was varied. In the third experiment, $d_{h}$ was kept constant $\left(d_{h}=29.2^{\prime}\right)$ and $d_{v}$ was varied. Displacements were kept well within the range of $0^{\prime}-30^{\prime}$ for smooth motion perception (Braddick, 1974; Chang \& Julezs, 1983). To avoid adaptation and learning effects where feasible, the directions for each of the two possible matches were randomized by selecting the sign of $d_{v}$ for each stimulus randomly.

The stimuli were generated on an Atari 1040 ST computer with a $2 \mathrm{MB}$ extended memory (so 64 frames could be stored as bit maps). An Atari SM125 high-resolution 70- $\mathrm{Hz}$ white phosphor monochrome monitor was used (luminance $71 \mathrm{~cd} / \mathrm{m}^{2}$ ). The monitor dimensions were $13.6 \times 21.7 \mathrm{~cm}(400 \times 640$ pixels $)$, and the monitor was viewed binocularly, with natural pupils, at a distance of $200 \mathrm{~cm}(1$ pixel $=$ $\left.0.58^{\prime}\right)$, which was well within the accommodation range.

In order to examine the effects of orientation and elongation of image elements on motion perception, we employed bar elements in the first three experiments. The bar elements (of variable length $L$, width $W$, and orientation $\alpha$ ), sketched in Figure 2A, were drawn in black on a white background, so that the visual system would adapt to a well-defined luminance level. In the fourth experiment, we used oriented disk elements. An oriented disk element is sketched in Figure 2B. It consists of a white center part, with a variable width $W$, superimposed on a black disk with a diameter $D=18.6^{\prime}$. The disk elements were drawn on a gray background (actually a checkerboard pattern, in which 1 check $=1$ pixel).

All experiments were done with fixation on a spot at the center of the screen and with frame displays lasting $168 \mathrm{msec}$ (four frames of $42 \mathrm{msec}$ ). Given this setup, one session of 100 stimuli lasted approximately $3 \mathrm{~min}$. Navon (1976) had already noted that durations of 150 msec were certainly sufficient for figural identification (although it did not bear on the motion perceived) and McKee and Nakayama (1984) had shown that a velocity discrimination task was almost optimal for such short durations.

Some side effects took place at the boundaries of the display, where image elements disappeared or had no matching partner. A disappearing element seemed to lead to perceptually confusing effects. Ramachandran and Anstis (1986) have shown that this conflict can be solved by providing occluding objects to hide the disappearing elements. Therefore, we covered our screen with a black circular mask that had a radius of 117'. At the borders of the mask, the image elements were thus perceived to move under the mask.
A

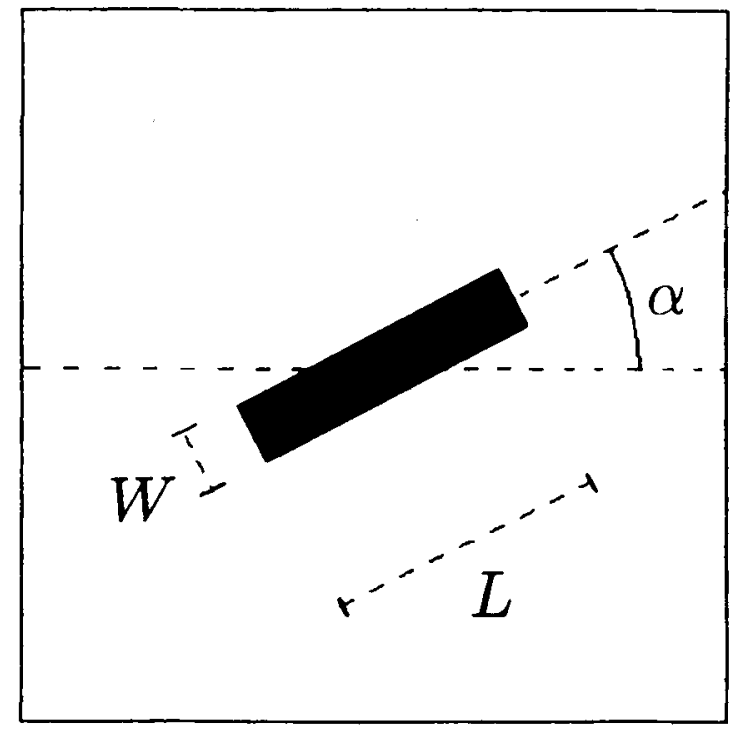

B

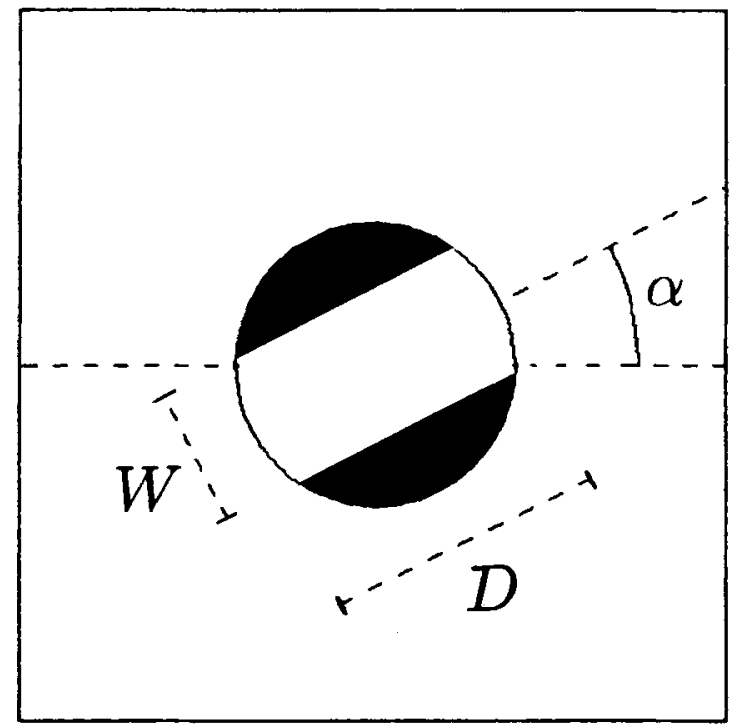

Figure 2. Sketch of the used elements. The orientation $\alpha$ of the elements is expressed counterclockwise with respect to the positive horizontal. (A) Bar elements of variable length $(L)$ and width $(W)$ were used in the first three experiments and are drawn on a white background. (B) Oriented disk elements (diameter $D$ ) were used in the fourth experiment and are drawn on a gray background. The width of the white center part of the disk element is $W$.

The resulting configurations for the first and second experiments are shown in Figure 3.

Six observers participated in the experiments, including two of the authors (H.S. and P.W.). For reasons of clarity, only the results of H.S. and P.W. are presented. H.S. is myopic $(-2.5 \mathrm{D})$ and P.W. is emmetropic. All observers had normal or corrected-to-normal 
A

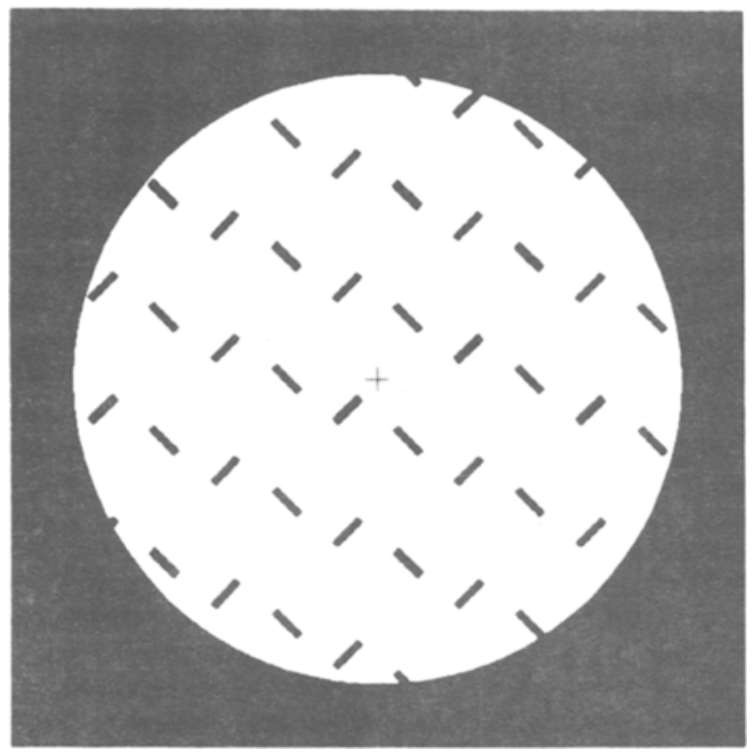

B

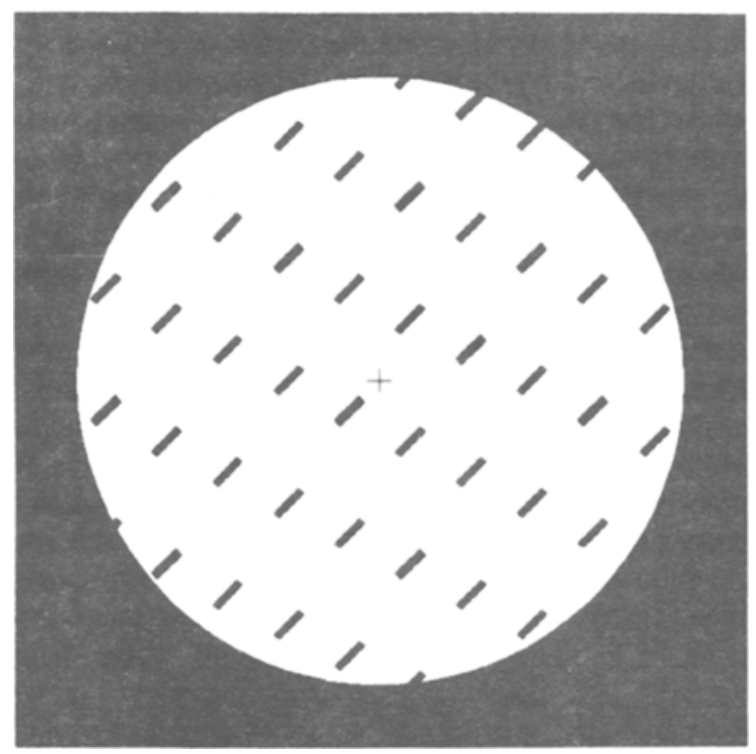

Figure 3. Schematic representation of two configurations used in different experiments: (A) differently oriented bar elements (oriented $\alpha$ and $-\alpha$ ) in the horizontal motion path (first experiment); (B) identical bar elements in both motion paths with orientation $\alpha$ (second experiment).

vision. The results were both qualitatively and quantitatively very similar for all observers.

\section{The Orientation Difference Between \\ Bar Elements}

In this first experiment, we examined the influence of the orientation difference between bar elements on apparent motion perception. The configuration is shown in Figure 3A. As has been mentioned in the Method section, the sum $d_{v}+d_{h}$ was kept constant. This had the ad- vantage that for an isotropic motion detection system (i.e., one having the same physical properties in all directions), the element velocities in the stimulus would be the same for $\alpha=0^{\circ}$ as for $\alpha=90^{\circ}$.

Image Elements $A$ and $B$ (see Figure 1) were bar elements, identical in length, width and flux. The two bar elements differed only in their orientations: $\alpha$ and $-\alpha$, respectively (the orientations are expressed in degrees counterclockwise with respect to the positive horizontal). Hence, Bar Elements A and B were mirror images of each other with respect to the vertical.

The curves in Figure 4 show the $R_{p s e}$ values (the ratio of vertical and horizontal distances, $d_{v}$ and $d_{h}$, at which horizontal and vertical motion perception were equally probable) as a function of orientation $\alpha$ for four bar lengths and two observers.

The displayed series of frames at orientation $\alpha$ was identical to the series at orientation $\left(180^{\circ}-\alpha\right)$, except that the frames were cyclically shifted. Therefore, we only varied $\alpha$ between $0^{\circ}$ and $90^{\circ}$; all curves were per definition symmetric around $\alpha=90^{\circ}$.

We found a pronounced effect of the bar element orientation on the ratio $R_{p s e}$. In particular, for large bar lengths ( $L=14.0^{\prime}$ and $\left.L=28.1^{\prime}\right), R_{\text {pse }}$ deviated noticeably from 1. At smaller bar lengths, the effect decreased but was still noticeable, although the size of the bar elements relative to their displacement per frame became very small.

A salient feature of all the curves was the fact that the ratio (which is a measure for dissimilarity between bar elements) reached its extremes where the orientation difference between bar elements disappeared ( $\alpha=0^{\circ}$ and $\alpha=90^{\circ}$ ). Another outstanding feature was that at $\alpha=45^{\circ}$ the bar elements were perpendicular to each other, whereas motion perception in both directions is almost equally probable in a configuration with $d_{v} \simeq d_{h}$.

It should be noted that, as in the experiments of Burt and Sperling (1981), and Ullman (1978, 1980), the orientation difference between bar elements was correlated with the relative orientation of each bar element with respect to its directions of possible motion. At $\alpha=45^{\circ}$, all bar elements were similar in their orientations with respect to motion direction $\left(45^{\circ}\right)$, and only a small deviation of $R_{p s e}(45)$ from 1 was observed. Moreover, at $\alpha=0^{\circ}$ and $\alpha=90^{\circ}$, all bar elements were equally oriented but differed maximally in their orientation with respect to the two possible motion directions.

This finding suggests strongly that orientation difference between bar elements does not influence the strength of motion perception between them. Secondly, it suggests that the orientation of a bar element with respect to its motion direction plays a dominant role in the strength of the motion perception.

The second experiment was an attempt to isolate the role of orientation with respect to motion direction.

\section{The Orientation of Bar Elements Relative to Motion Direction}

This experiment was almost identical to the first experiment, except that all image elements were identical in 
A

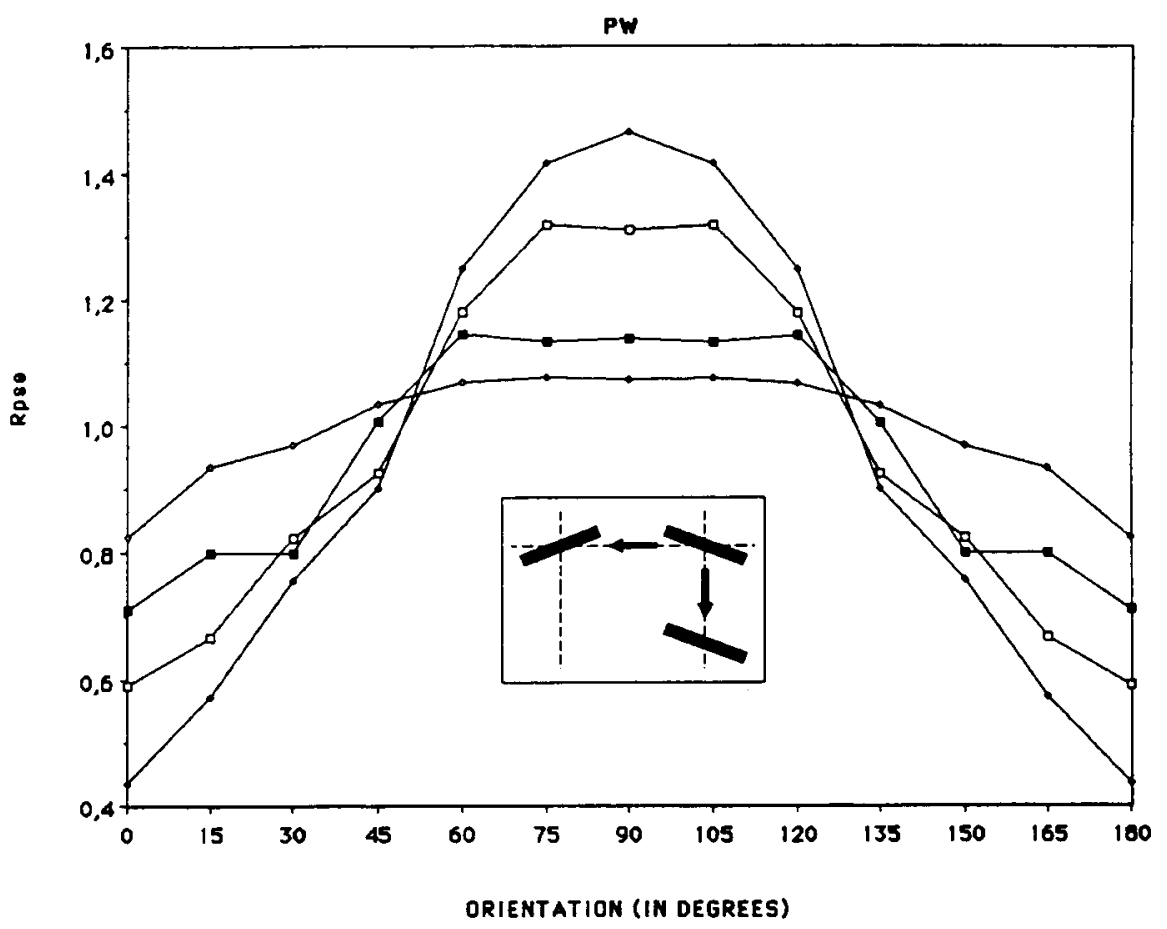

B

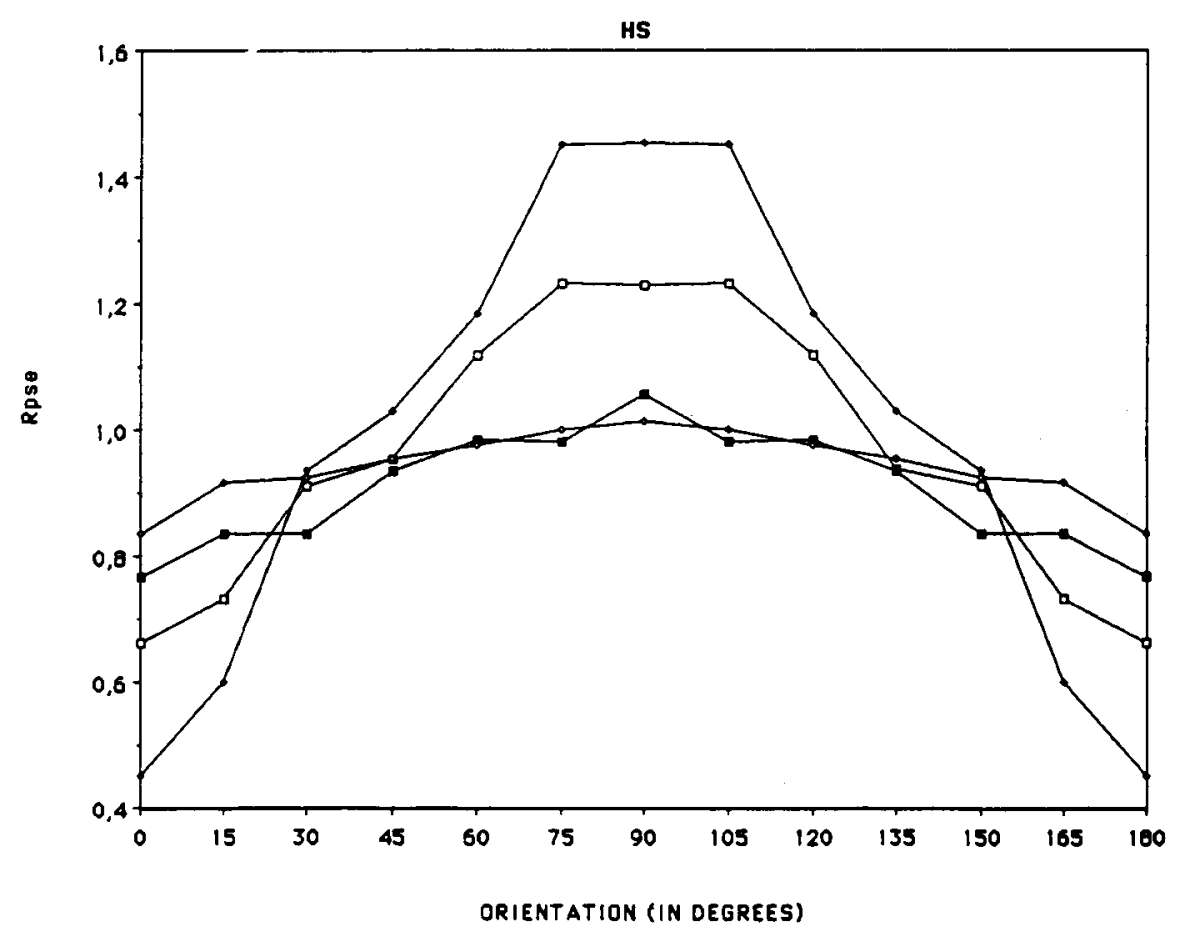

Figure 4. Distance ratio ( $\left.R_{p s e}\right)$ at which the probabilities of vertical motion perception between identical bar elements (oriented $\alpha$ with respect to the horizontal) and of horizontal motion perception between nonidentical bar elements (oriented $\alpha$ and $-\alpha$ ) in a motion path are equal (see inset). All curves are per definition symmetric around $\alpha=90^{\circ}$. $R_{p s e}$ values are plotted as a function of $\alpha$ for various bar lengths $(L)$ and two observers (P.W. and H.S.). $\diamond, L=3.5^{\prime} ; \bullet, L=7.0^{\prime} ; \square, L=14.0^{\prime}$; $\bullet, L=28.1^{\prime}$. 
orientation (see Figure 3B). The parameter varied was the orientation $\alpha$ of the bar elements relative to the horizontal.

The two competing motion paths differed only in the orientation of the bar elements of the path with respect to motion direction. If motion perception strength is governed only by orientation differences between elements in a motion path, one may naively expect for this experiment that $R_{p s e}(\alpha)=1$.

The results (Figure 5) lead to the following observations:

1. At $\alpha=45^{\circ}$, Bar Elements A and B are identical in orientation with respect both to each other and to the possible motion directions. The ratio $R_{p s e}(45)$, however, deviates slightly though markedly from 1 for all observers. Furthermore, if motion perception were isotropic, one would expect $R_{p s e}(45-\beta)$ to be the reciprocal of $R_{p s e}(45+\beta)$ for $0<\beta<45^{\circ}$. The data do not bear this prediction out, however.

2. At $\alpha=0^{\circ}$, all bar elements are horizontally oriented. Here $R_{p s e}$ decreases dramatically. The vertical distance has to be decreased relative to the horizontal distance almost by a factor of 2 to make both of the possible motion directions equally probable perceptually. An analogous result holds at $\alpha=90^{\circ}$, where all bar elements are vertically oriented and the horizontal distance has to be decreased relative to the vertical distance almost by a factor of 2 to make both of the possible motion directions equally probable perceptually. Both of these results demonstrate the dominance of motion perception in the direction of the orientation of the bar elements.

3. The global form of the curves in Figure 5 is almost identical to the form of the curves in Figure 4. To illustrate this, we have plotted the $\boldsymbol{R}_{\text {pse }}$ values at one bar length $\left(L=28.1^{\prime}\right)$ for both identically oriented (Figure 5) and differently oriented elements (Figure 4) in Figure 6. An orientation difference between bar elements in the horizontal motion path induces a systematic but small increase of the $\boldsymbol{R}_{p s e}$ values. This means that the orientation difference between the bar elements in the horizontal motion path slightly increases the dominance of the vertical motion path.

This global observation shows clearly that orientation differences between bar elements in a motion path play a secondary role in the selection of motion paths, in comparison with the influence of the bar element orientation with respect to the motion direction.

4. The overall spatial structure of the pattern (the arrangement of the elements in diagonal rows) is accentuated when the bar elements are oriented along the diagonal row. Since all elements in this experiment were identically oriented, the results were expected to be biased by the orientation of the bar elements with respect to the orientation of the diagonal rows.

When $d_{v}=d_{h}$, the perceptual appearance of diagonal rows is much stronger at element orientation $\alpha=45^{\circ}$ (oriented along the diagonal row) than at element orientation $\alpha=135^{\circ}$ (oriented perpendicularly to the diagonal row). Effects of overall spatial structure would lead to an asymmetry of the curves of Figure 5 around $\alpha=90^{\circ}$.
Although this asymmetry can be observed (Figure 5), it is fractional.

5. At increasing bar length, a growing effect of element orientation with respect to motion direction is observed (Figure 5). This already suggests that the elongation of the bar elements (length/width ratio) was an important parameter in these experiments.

In the third experiment, we examined the role of elongation with respect to the strength of motion perception.

\section{The Influence of Bar Length and Width for Identical Horizontal Bar Elements}

Whatever motion detection mechanism might have been involved, from the second experiment it is obvious that this mechanism is sensitive to elongation in the direction of its velocity tuning direction. This raised the question of how the output of such a mechanism might depend on the elongation and size of the motion-eliciting bar elements relative to the receptive size of the motion detection mechanism.

Therefore, the horizontal displacement $d_{h}$ of the bar elements was fixed in the third experiment $\left(d_{h}=29.3^{\prime}\right)$. The vertical displacement $d_{v}$ was varied. As in the second experiment, identical bar elements were used (Figure 3B). However, in the present experiment, the element orientation was kept horizontal $\left(\alpha=0^{\circ}\right)$ and bar width and bar length were varied.

Figure 7 shows the influence of bar length and bar width for identical horizontal bar elements on apparent motion perception. At a constant width $(W)$, an increase in bar length $(L)$ of up to $L=d_{h}$ causes a very significant decrease of $\boldsymbol{R}_{\text {pse }}$. Furthermore, at a constant bar length, $R_{p s e}$ is found to increase monotonically with bar width (as far as can be judged from four width values). The curves are systematically shifted along the $\boldsymbol{R}_{p s e}$ axis at increasing bar width.

Due to the good reproducibility of these measurements, we can observe a strikingly similar fine structure for all curves and all subjects. Local maxima occur at bar lengths $L$ that nearly are multiples of the horizontal displacement $d_{h}$; that is, $L=n d_{h}(n=1,2,3)$. A similar but informal experiment at the horizontal shift $d_{h}=18.7^{\prime}$ also showed local maxima at $n d_{h}(n=1,2,3)$. At these bar lengths, an element of frame $f_{n}$ will start to overlap with the horizontally displaced elements in frames $f_{n+1}, f_{n+2}$ and $f_{n+3}$, respectively. The physical overlap of successive elements in time introduces local contrast effects that may bias motion perception.

As a test of this claim, we carried out a similar experiment, but eliminated physical overlap by a small element orientation $\alpha=5^{\circ}$ (Figure 8A). The local maxima almost vanish and the curve levels off for large bar lengths. However, the local maximum at $d_{h}$ remains, indicating other effects still existing. Thus, physical overlap of the bar elements in time can only partly explain the local maxima. Moreover, in a similar experiment but with elements oriented $5^{\circ}$ in the other direction $\left(\alpha=-5^{\circ}\right)$, the local maxima survived (also Figure 8A). Local form differ- 
A

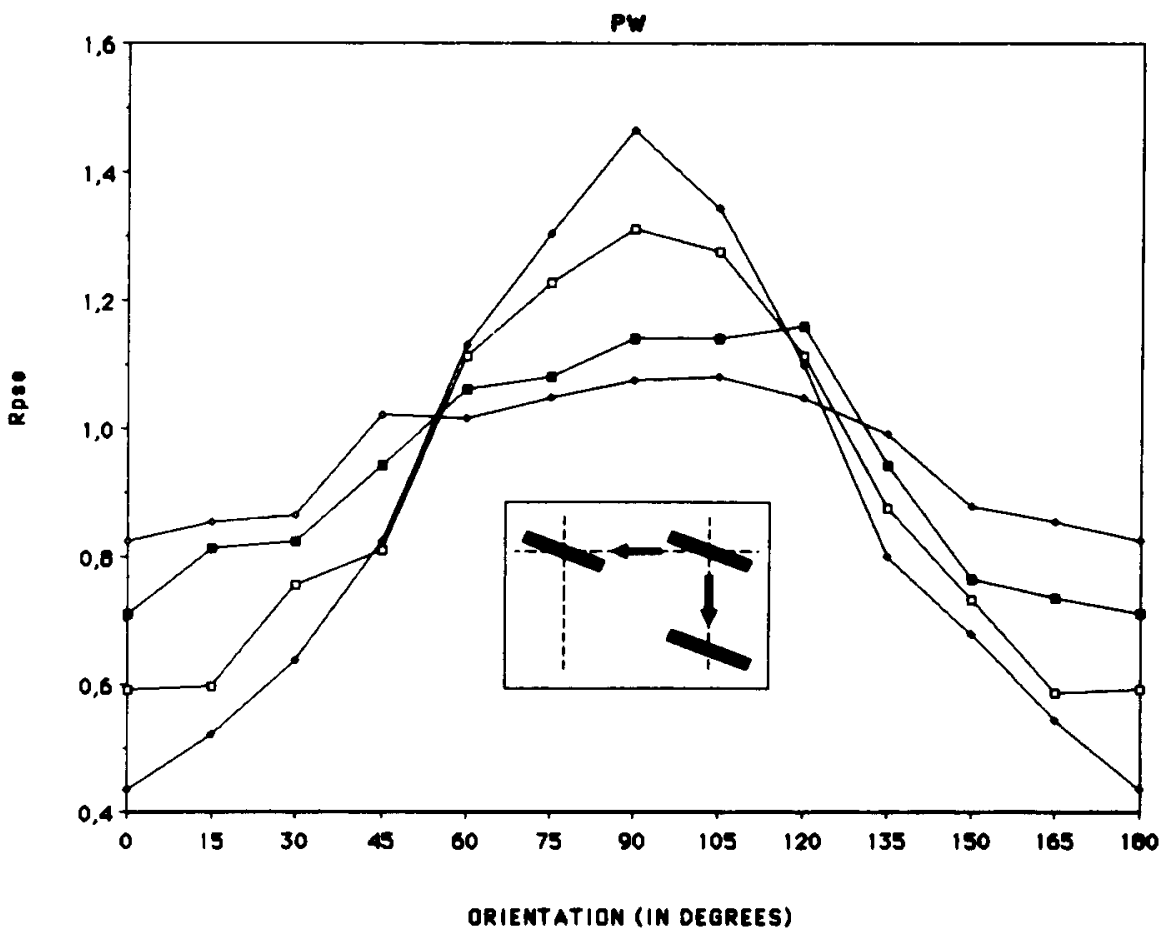

B

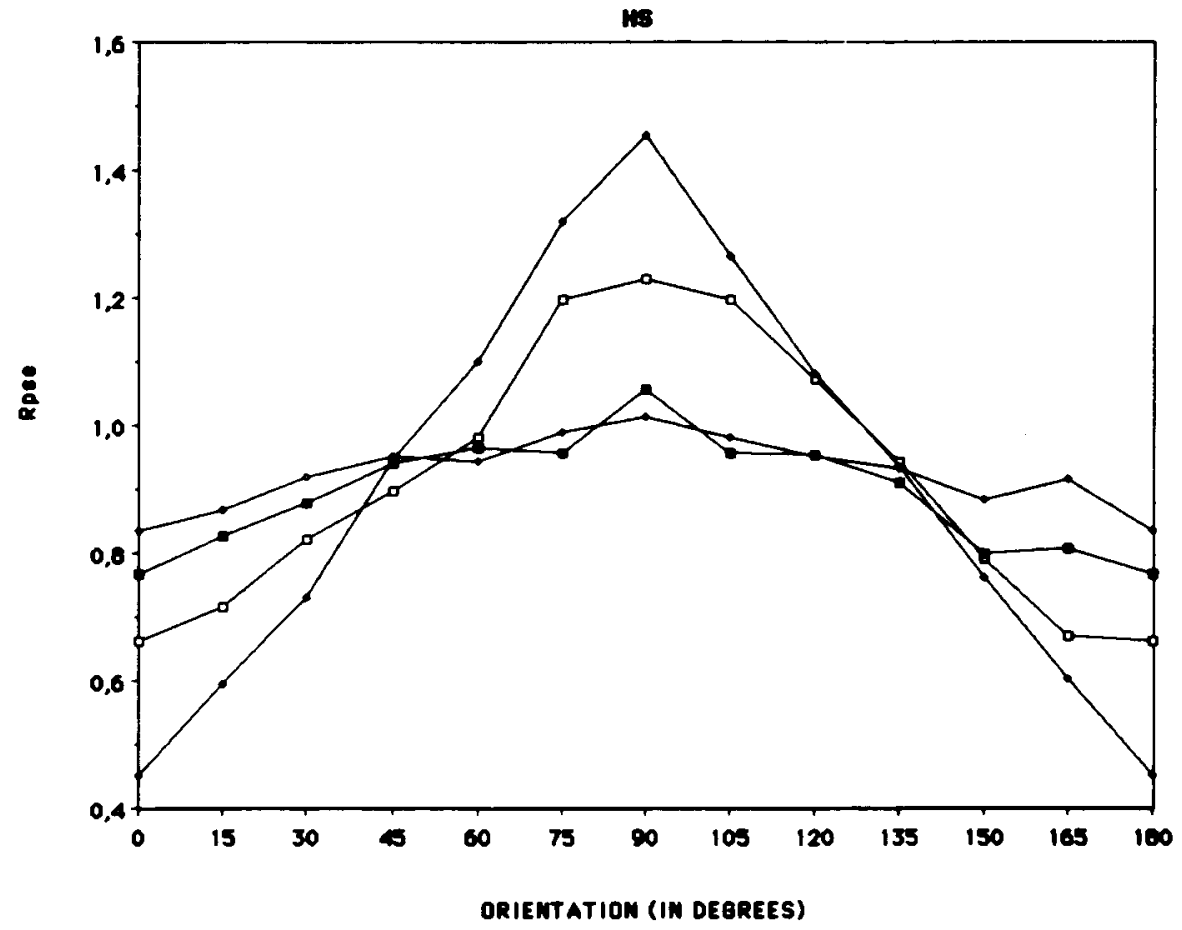

Figure 5. Distance ratio $\left(R_{p s e}\right)$ at which the probabilities of vertical and horizontal motion perception are equal. All bar elements have identical orientations $\alpha$ (vertical and horizontal motion paths differ only in their element orientation with respect to motion direction; see inset). $R_{p s e}(0)$ and $R_{p s e}(90)$ are taken from Figure $4\left[R_{p s e}(180)=R_{p s e}(0)\right]$. $R_{p s e}$ values are plotted as a function of $\alpha$ for various bar lengths $(L)$ and two observers (P.W. and H.S.). $\diamond, L=3.5^{\prime} ; \square, L=7.0^{\prime} ; \square, L=14.0^{\prime}$; $\bullet, L=28.1$ '. The bar width $(W)$ is always $1.2^{\prime}$. 
A

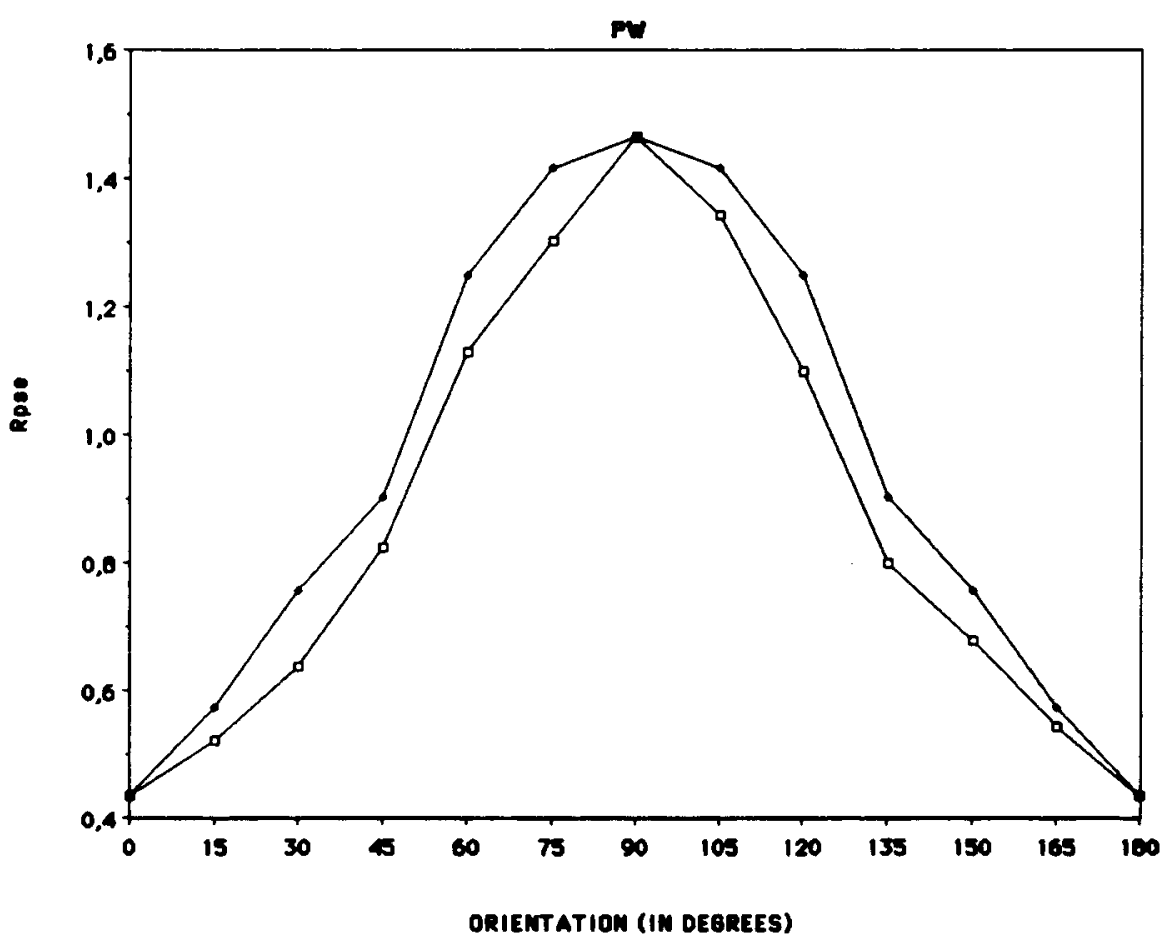

B

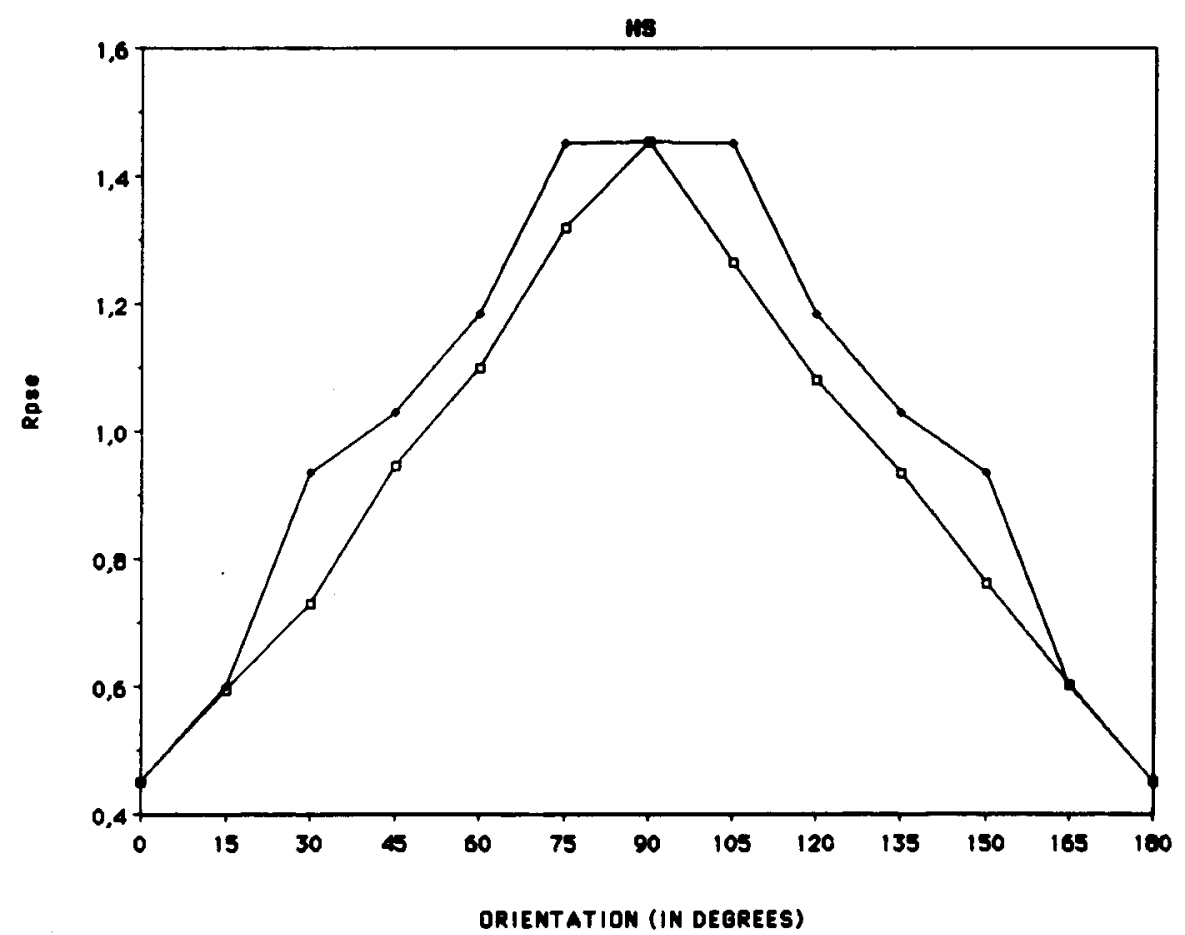

Figure 6. $R_{p s e}$ values for bar length $L=28.1^{\prime}$ and bar width $W=1.2^{\prime}$, as a function of orientation $\alpha$. The curves are taken from Figures 4 and 5. $\square$, identically oriented bar elements $(\alpha)$; $\diamond$, differently oriented bar elements ( $\alpha$ and $-\alpha$ ) for both observers (P.W. and H.S.). 
A

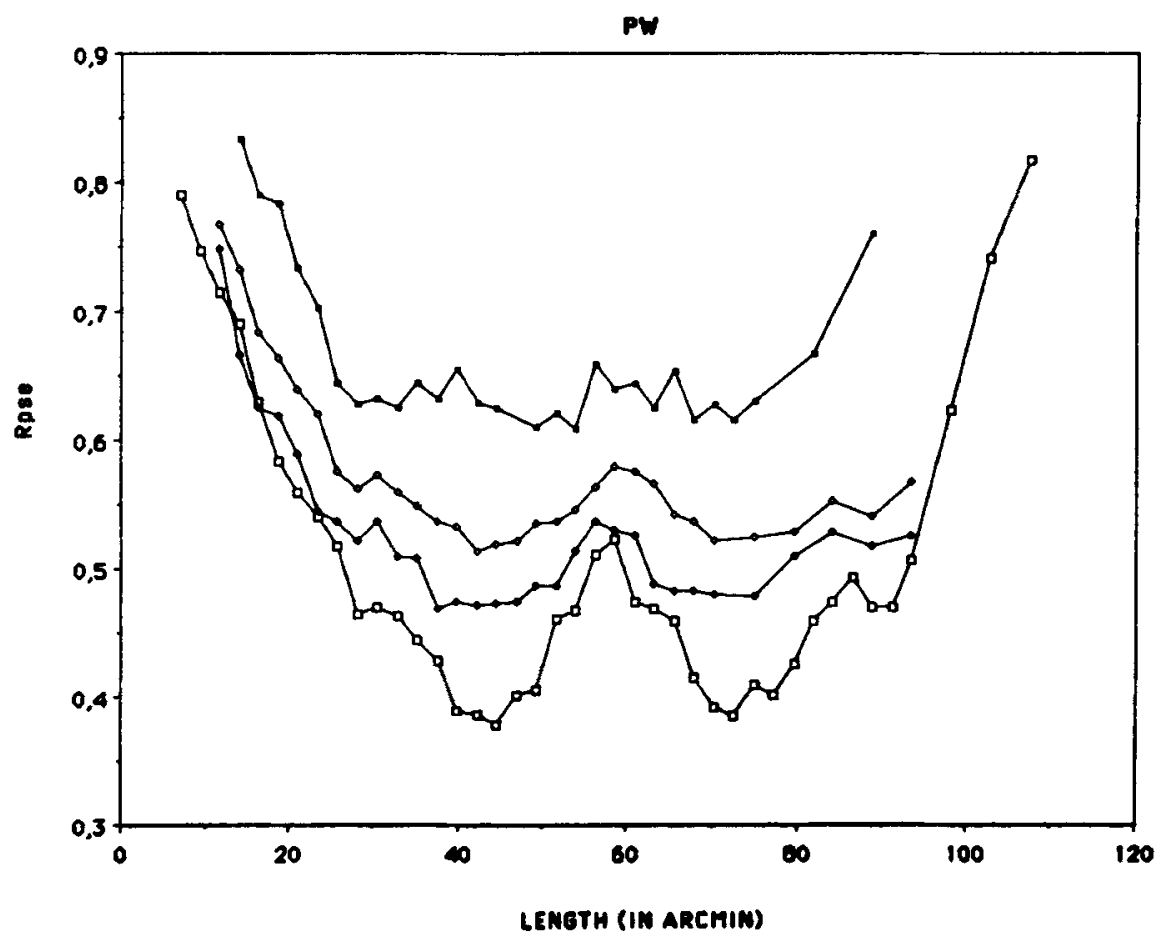

B

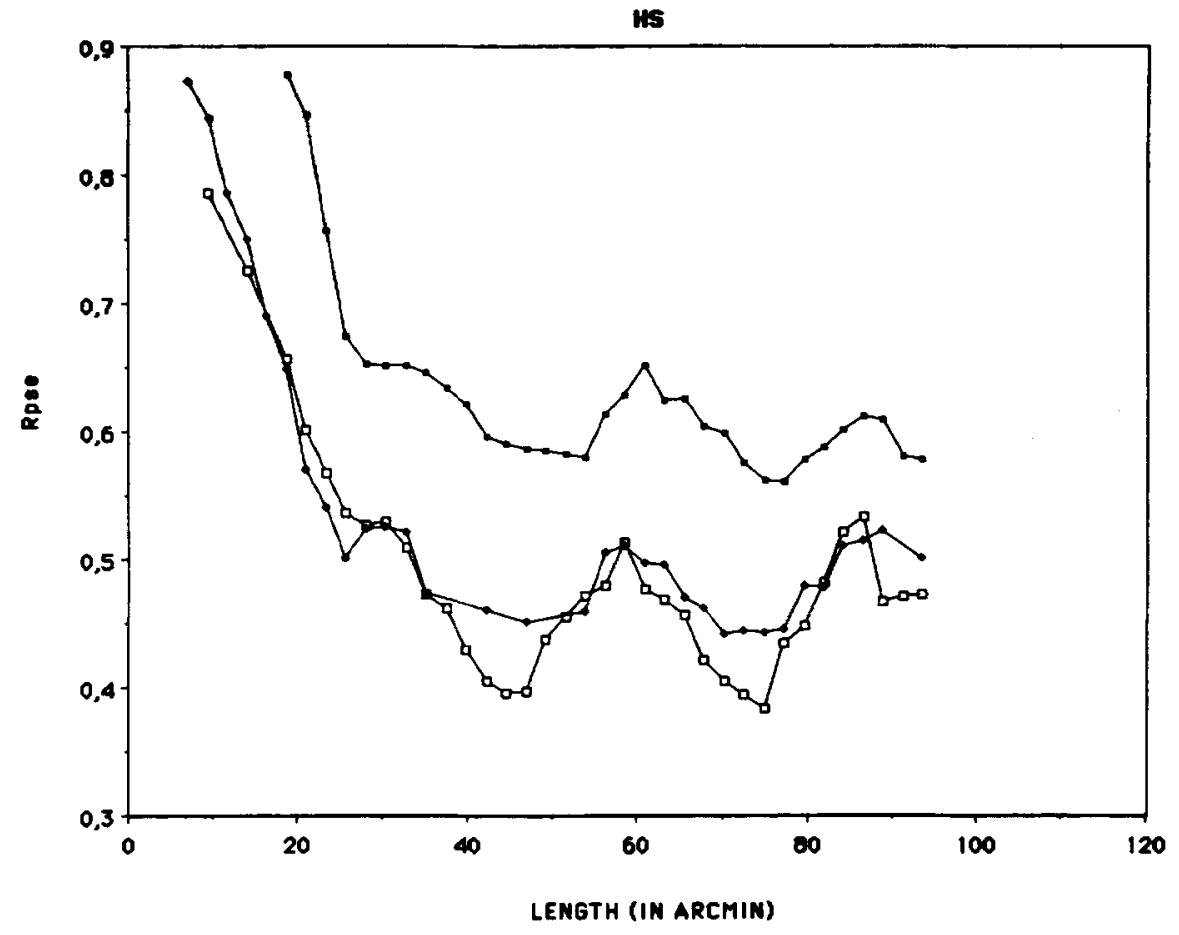

Figure 7. Distance ratio ( $R_{p s e}$ ), at which the probability of vertical motion perception (between horizontal bar elements) is equal to the probability of horizontal motion perception (between horizontal bar elements), as a function of bar length $(L)$. $R_{p s e}$ values are plotted for several bar widths $(W)$ and two observers (P.W. and H.S.). $\square, W=1.2^{\prime} ; \diamond, W=3.5^{\prime} ; \diamond, W=8.2^{\prime} ; \square, W=14.0^{\prime}$. 
A

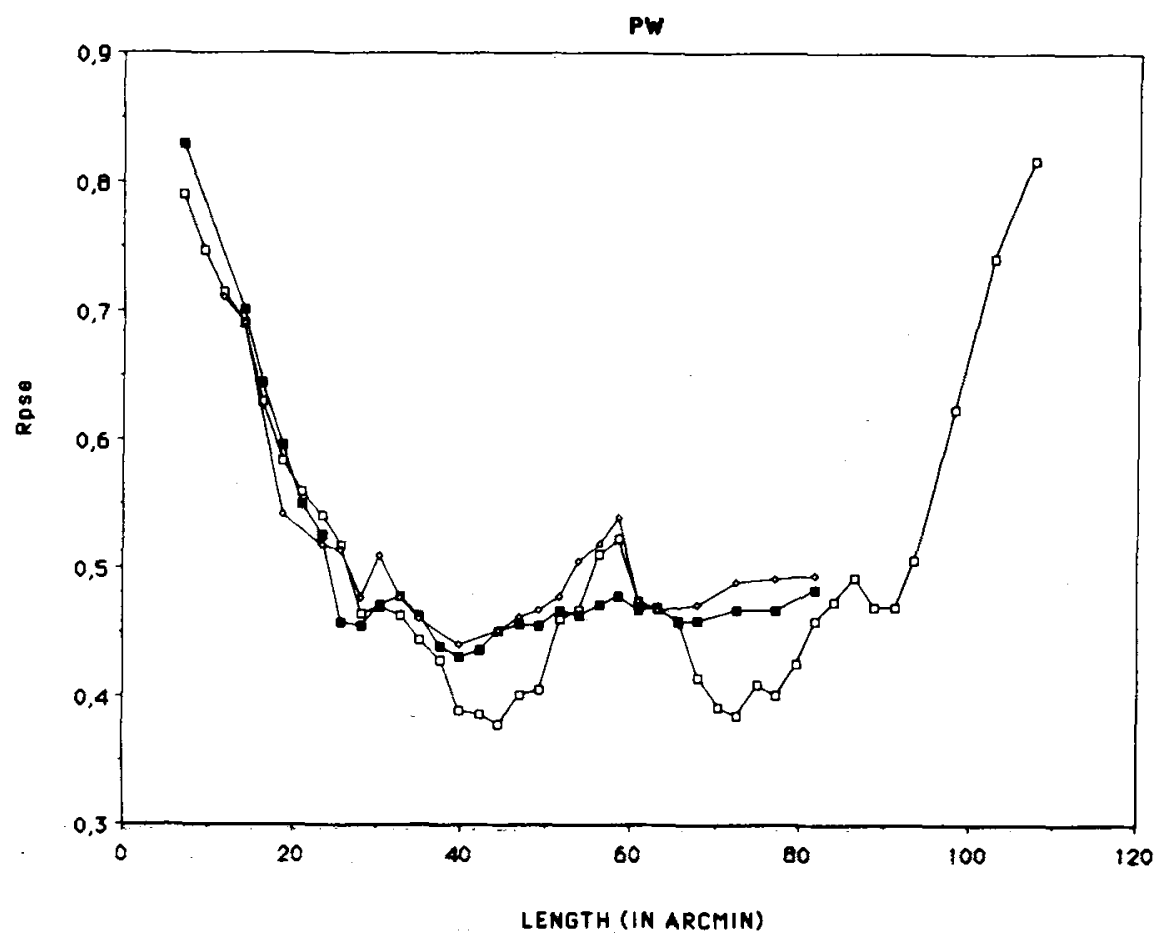

B

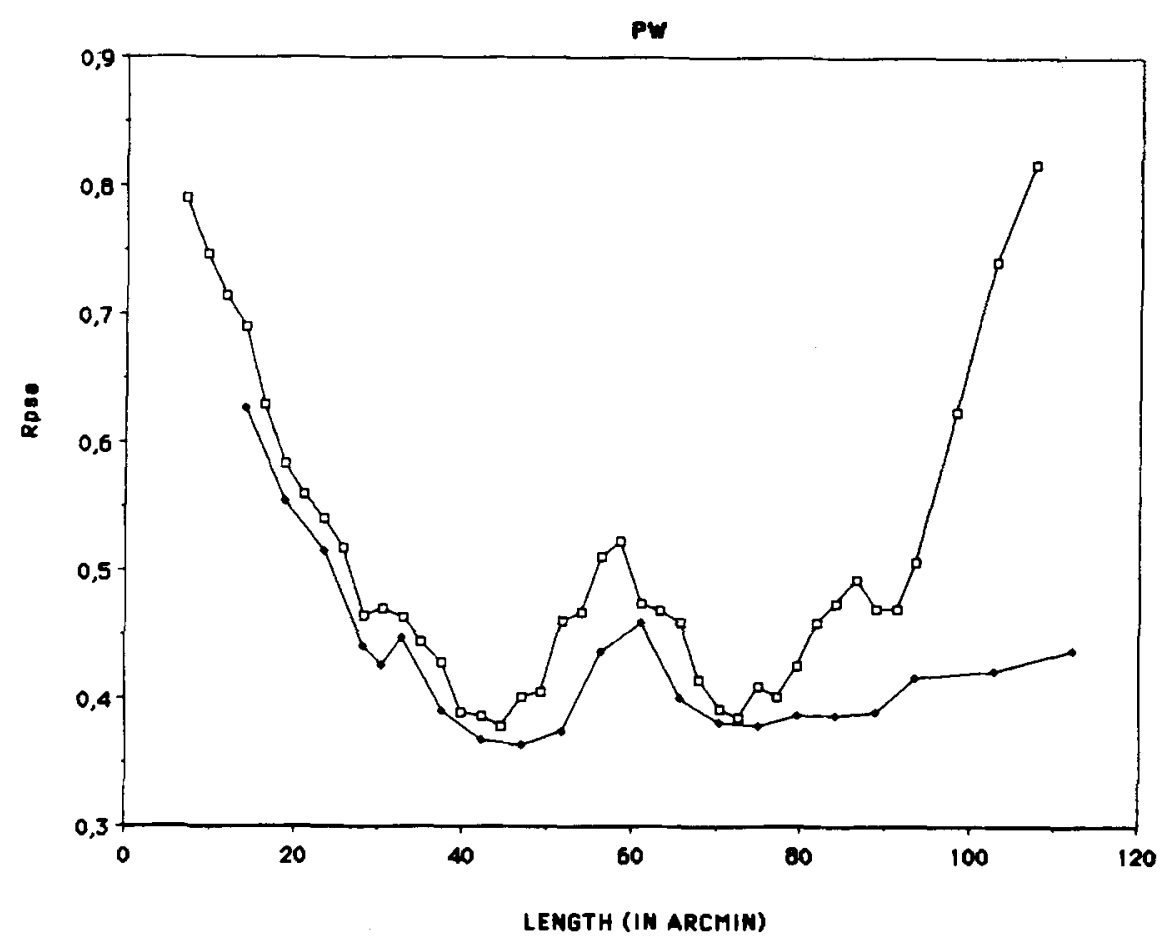

Figure 8. Distance ratios are plotted for identically oriented bar elements as a function of bar length $(L)$. The bar width $(W)$ is always $1.2^{\prime}$. (A) Curves for several orientations $\left(\square, \alpha=5^{\circ}\right.$; $\left.\vartheta, \alpha=-5^{\circ}\right)$. The curve with open squares is taken from Figure 7 as a reference $\left(\alpha=0^{\circ}\right)$. (B) Curves for horizontal bar elements but different horizontal interdiagonal distances $\left(\square, 117^{\prime} ; \bullet, 234^{\prime}\right)$. 
ences are identical for both orientations, indicating that the local maxima of the curves essentially depend on the orientation of the elements relative to the diagonal.

For bar lengths close to the horizontal distance between two neighboring diagonal rows in one frame, the bar elements are nearly contiguous in one frame. The interspaces of the bar elements start to dominate perceptually as image elements. The increase at the tail of the curves in Figure 7 can therefore be explained by the form change of the interspaces, and it should be considered as an artifact. This effect can be eliminated by reducing the spatial density of the diagonals by a factor of two, as can be observed in Figure 8B.

However, Figure 8B still reveals conspicuous extrema at $d_{h}$ and $2 d_{h}$, which provides evidence that the local maxima are not an artifact caused by the periodicity of the diagonals.

\section{Unconfounding Separation and Orientation}

While the displacements of the elements $\left(d_{v}, d_{h}\right)$ are always well controlled in our experiments, the spatial separation (the minimum distance separating the closest points of two elements in a motion path) is not. The fact that the manipulation of element orientation and element length in the first three experiments was correlated with the spatial separation of the elements makes an alternate explanation of the results possible.

An alternate explanation can be made simply in terms of a nearest neighbor rule: Motion is seen preferentially, between the bar elements with the smallest separation. One way of isolating the effect of orientation is to replace the bar elements with oriented disk elements (see Figure 2B), whose separation is unaffected by the manipulation of orientation.

Therefore, the first two experiments were repeated with the oriented disk elements. The diameter $(D)$ of the disk element was kept constant $\left(D=18.6^{\prime}\right)$. The orientation $\alpha$ and the width of the white center part $W$ of the disk element were varied.

First we varied the width $(W)$ of the white center part of the disk elements and measured $R_{p s e}$ for identical, horizontally oriented elements. The results are shown in Figure 9 for both observers.

At $W=0^{\prime}$ and $W=D=18.6^{\prime}$ (element diameter), the elements were, respectively, black and white disks without an orientation. At these widths, the measured $R_{p s e}$ is an indication of the anisotropy of the motion detection system (see below, next section). However, for values of $W$ between $0^{\prime}$ and the element diameter $(D)$, we can expect effects of orientation. We find a strong dominance of the motion path along the disk element orientation that is maximal at $W=8.7^{\prime}$ for Observer P.W. and at $W=11.0^{\prime}$ for Observer H.S.

It should be noted that perceptual velocity is much slower and motion less smooth in both vertical and horizontal directions at a width of $W=7^{\prime}$, which is close to the width $W=7.4^{\prime}$ at which the elements are equiluminant to the gray background.

At the optimal width of the center part (P.W., $W=8.7^{\prime}$; H.S., $W=11.0^{\prime}$ ), we measured $R_{p s e}$ as a function of the

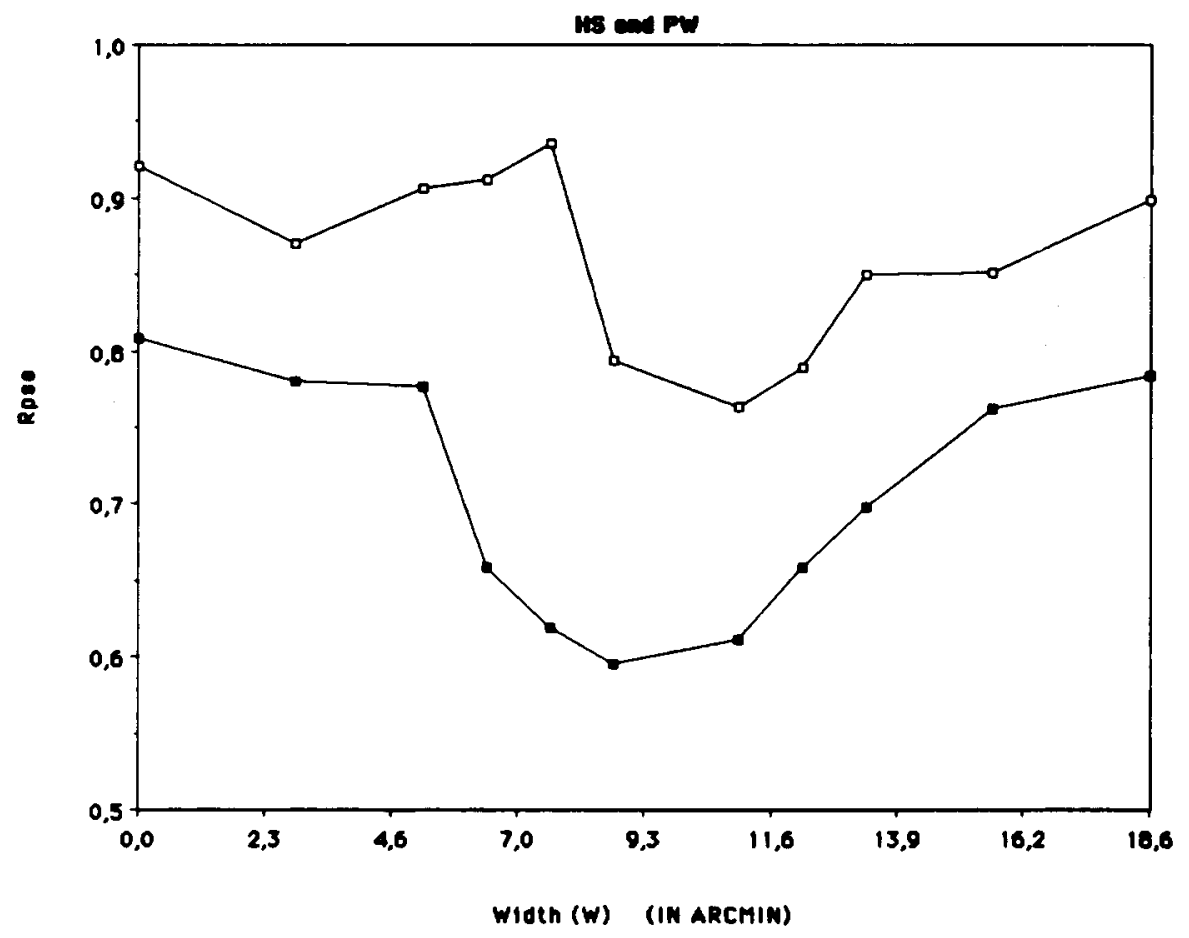

Figure 9. $R_{p s e}$ values for identical horizontally oriented disk elements (diameter $D=18.6^{\prime}$ ) as a function of the width $(W)$ of the white center part of the disk element (see Figure 2B). $\square, \mathrm{Ob}$ server H.S.; ", Observer P.W. 
orientation $\alpha$ for both observers. Just as in Figure 6, we plotted the $R_{p s e}$ values for both identically oriented $(\alpha, \alpha)$ and differently oriented disk elements $(\alpha,-\alpha)$ in one figure (Figure 10).

The similarity of the results in Figure 10 to the results in Figure 6 is obvious. Although spatial separation is constant for varying orientation, a clear preference for motion along element orientation remains. The effect of orientation is even stronger than the effect that can be expected for bar elements (see above, in the section Orientation of Bar Elements) with the same dimensions as the white center part of the disk element. For example (for Observer P.W.), a white center with dimensions $8.2^{\prime} \times 18.6^{\prime}$ yielded a lower $R_{p s e}$ of 0.60 (see Figure 10) than did bar elements with similar dimensions $\left(8.7^{\prime} \times 18.6^{\prime}\right)$, which yielded a $R_{p s e}$ of 0.68 (see Figure 7 ).

It should be noted that, for Observer P.W., $R_{p s e}$ is systematically higher for identically oriented elements $(\alpha, \alpha)$ than for differently oriented elements $(\alpha,-\alpha)$, indicating that motion between differently oriented elements is systematically stronger than motion between identically oriented elements.

From the results of this experiment, we conclude that the observed preference for motion along the element orientation in this experiment was caused by the effects of orientation with respect to motion direction.

Supported by these results, we assume that the preference for motion along the element orientation found in the second experiment can be ascribed primarily to the same effects of orientation with respect to motion direction, and that the confounding effect of separation may be small or even nonexistent.

\section{Unconfounding Anisotropy and Orientation}

Our results show that the $R_{p s e}$ value for a configuration with a horizontal and vertical motion path differs significantly from the reciprocal $R_{\text {pse }}^{\prime}$ value for a similar configuration with horizontal and vertical motion paths interchanged. A systematic preference exists for horizontal motion paths. It is not clear whether this was due to a geometrical scaling of our visual field, or that this effect derives from a decision stage at which horizontal and vertical motion were compared. Motion perception is not invariant under rotation; in what follows, we will try to quantify this anisotropy.

Here we will naively introduce anisotropy by assuming that our visual system uses different "yardsticks" for measuring vertical and horizontal distances. Suppose that for an isotropic visual system $\boldsymbol{R}_{p s e}=r$. The values $\boldsymbol{R}_{\text {pse }}$ (for a certain configuration) and $R_{p s e}^{\prime}$ (for the rotated configuration) in the eyes of an anisotropic visual system (which scales vertical distance by a factor $h$ with respect to horizontal distance) will be:

$$
R_{p s e}=\frac{r}{h} \text { and } R_{p s e}^{\prime}=\frac{1}{r h},
$$

so that

$$
h=\frac{1}{\sqrt{R_{p s e} R_{p s e}^{\prime}}} \text { and } r=\sqrt{\frac{R_{p s e}}{R_{p s e}^{\prime}}} .
$$

This putative relation provides a means to correct our data for anisotropy. We will compute the scale factor $h$ and the measure for the influence of form on motion perception after correction for anisotropy $(r)$ for several experiments, to check the validity of the scaling assumption.

From the second experiment, we obtain $R_{p s e}(\alpha)$ and $R_{p s e}^{\prime}=R_{p s e}\left(90^{\circ}-\alpha\right)$, for $\alpha=0^{\circ}, 15^{\circ}, 30^{\circ}$, and $45^{\circ}$. Substituting these $R_{p s e}$ and $R_{p s e}^{\prime}$ in Equation 1, we obtain the corrected distance ratio $r$, for the homogeneous paths of elements oriented $\alpha$ degrees with respect to motion direction, and the scale factor $h$. We present $h$ and $r$ for several orientations and bar lengths in Table 1 .

The anisotropy index $(h)$ increases systematically as bar length increases, for both observers. The dependency of $h$ on orientation is not systematic and is small. A common conclusion is that $h$ depends on bar length and slightly on orientation. This cannot be expected from a linear geometrical transformation of space. One can speculate that anisotropy is caused by small differences between the density of neurons tuned for vertical motion and the density of neurons tuned for horizontal motion. Moreover, densities can be different for subpopulations tuned to different spatial structures that move with different velocities.

\section{Discussion}

Our paradigm makes it feasible to assess feature selectivity and to quantify the characteristics of the human motion detection system very precisely. The results provide constraints for theories of apparent motion detection (Sperling et al., 1985).

These results imply that orientation relative to motion direction is an important feature of image elements. The dominance of a motion path is a monotonic decreasing function of the tilt of bar elements relative to the motion direction; it is optimal when the bar elements move along their orientation axis. Compared with this effect, the influence of orientation differences between bar elements in a heterogeneous motion path is weak. The same conclusion holds for the oriented disk elements, which we used to unconfound the effect of orientation with the minimum separation between elements. The results confirm the general predictions of Navon (1976) and Kolers (1972) that relatively simple aspects of form (orientation and elongation) can effect apparent motion perception.

With a method that differs from ours, Ullman (1980) reached the contradictory conclusion that orientation differences between bar elements in a heterogeneous motion path strongly affect apparent motion perception. For small orientation differences of $10^{\circ}$, he found distance ratios of 1.5 up to ratios of 5 for a $30^{\circ}$ orientation difference (at bar length $L=24^{\prime}$ and corresponding shifts). Moreover, at orientation differences exceeding $30^{\circ}$, identical elements were matched even when nonidentical elements were successively superimposed. Although orien- 
A

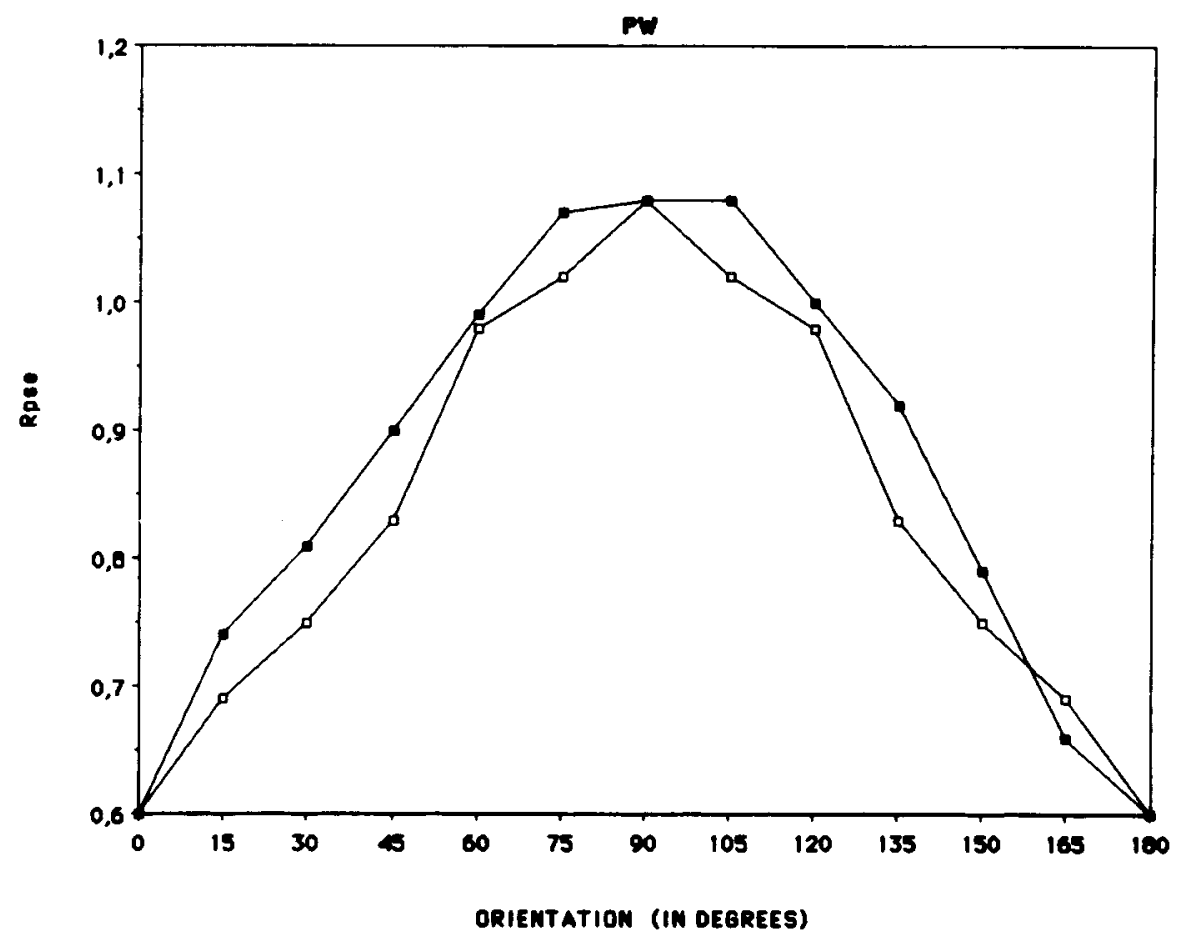

B

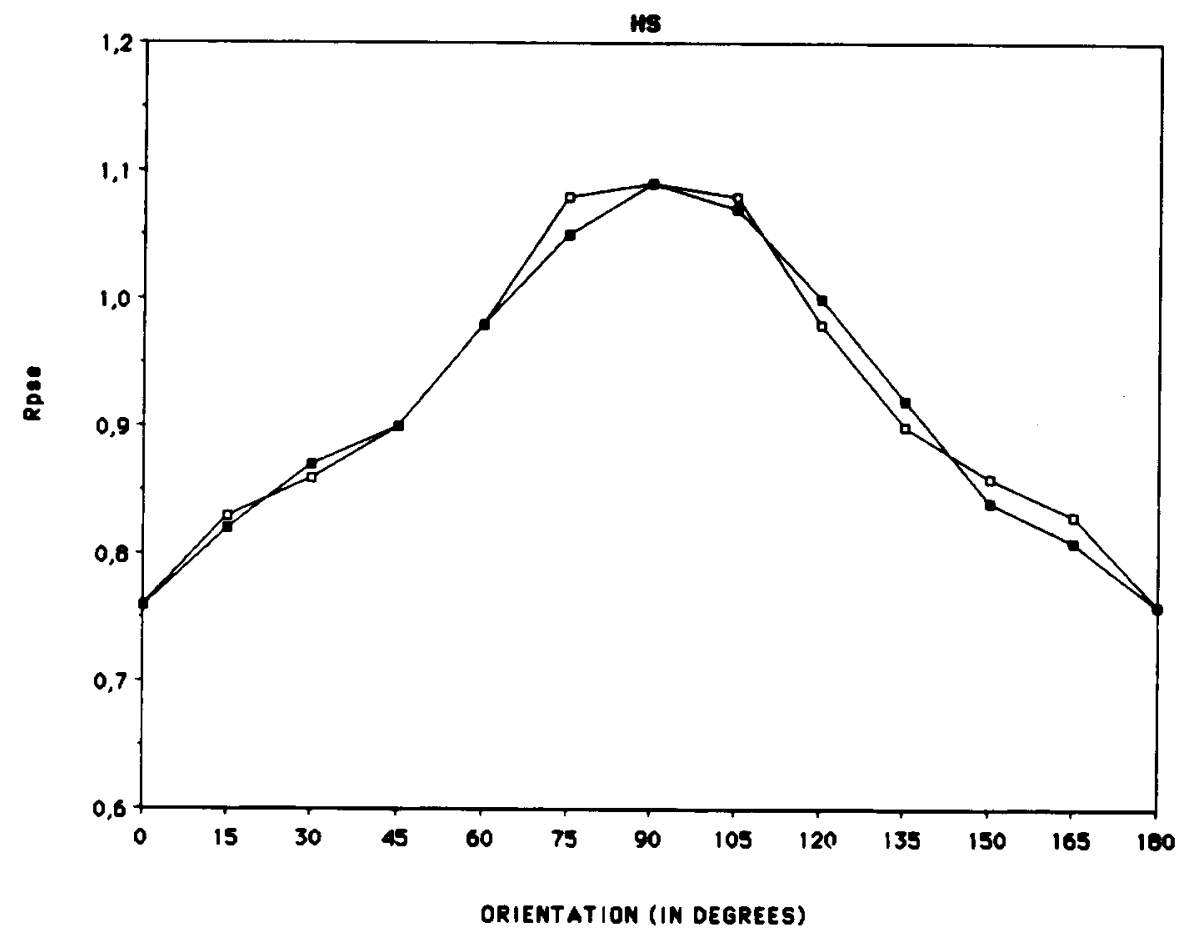

Figure 10. $R_{p s e}$ values for oriented disk elements (diameter $D=18.6^{\prime}$ ), as a function of their orientations $(\alpha)$. $a$, identically oriented bar elements $(\alpha)$; $\square$, differently oriented bar elements ( $\alpha$ and $-\alpha)$. The width $(W)$ of the white center part of the element is 8.7' for Observer P.W. and 11.0' for Observer H.S. 
Table 1

Anisotropy Index $(h)$ and Corrected $R_{p s e}$ Values $(r)$ for Several Orientations $(\alpha)$ and Lengths $(L)$ of Bar Elements for Two Observers

\begin{tabular}{|c|c|c|c|c|c|}
\hline \multirow[b]{3}{*}{$L$} & \multirow[b]{3}{*}{$\alpha$} & \multicolumn{4}{|c|}{ Observer } \\
\hline & & \multicolumn{2}{|c|}{ P.W. } & \multicolumn{2}{|c|}{ H.S. } \\
\hline & & $r$ & $h$ & $r$ & $h$ \\
\hline \multirow[t]{4}{*}{$3.5^{\prime}$} & $0^{\circ}$ & 0.88 & 1.06 & 0.91 & 1.09 \\
\hline & $15^{\circ}$ & 0.90 & 1.06 & 0.94 & 1.08 \\
\hline & $30^{\circ}$ & 0.92 & 1.07 & 0.99 & 1.07 \\
\hline & $45^{\circ}$ & 1.00 & 1.02 & 1.00 & 1.05 \\
\hline \multirow[t]{4}{*}{$7.0^{\prime}$} & $0^{\circ}$ & 0.79 & 1.11 & 0.85 & 1.11 \\
\hline & $15^{\circ}$ & 0.88 & 1.09 & 0.85 & 1.13 \\
\hline & $30^{\circ}$ & 0.88 & 1.07 & 0.85 & 1.09 \\
\hline & $45^{\circ}$ & 1.00 & 1.06 & 1.00 & 1.06 \\
\hline \multirow[t]{4}{*}{$14.0^{\prime}$} & $0^{\circ}$ & 0.67 & 1.14 & 0.73 & 1.11 \\
\hline & $15^{\circ}$ & 0.70 & 1.17 & 0.77 & 1.08 \\
\hline & $30^{\circ}$ & 0.83 & 1.09 & 0.92 & 1.11 \\
\hline & $45^{\circ}$ & 1.00 & 1.24 & 1.00 & 1.11 \\
\hline \multirow[t]{4}{*}{$28.1^{\prime}$} & $0^{\circ}$ & 0.55 & 1.25 & 0.56 & 1.24 \\
\hline & $15^{\circ}$ & 0.63 & 1.21 & 0.67 & 1.13 \\
\hline & $30^{\circ}$ & 0.75 & 1.18 & 0.82 & 1.12 \\
\hline & $45^{\circ}$ & 1.00 & 1.22 & 1.00 & 1.06 \\
\hline
\end{tabular}

tation differences and tilt were correlated in Ullman's experiment, we did not find such large effects (not even for orientation differences of $90^{\circ}$; see the result for $\alpha=45^{\circ}$ in Figure 4). The effect of orientation differehces has no potency at all for disabling the heterogeneous motion path in our experiments. This apparent inconsistency is probably caused by the fact that Ullman used only two frames, one row of bar elements, and entirely different temporal parameters ( $300 \mathrm{msec}$ per frame, and an interstimulus interval of $20 \mathrm{msec}$ ).

Burt and Sperling (1981) derived a precise tradeoff function between the displacement of image elements and the time interval of displacement, and they proved the existence of an optimal time interval for maximizing the strength for any path, which was affected only slightly by figural details of the stimulus elements (e.g., orthogonally oriented bar elements). Their finding that motion between perpendicularly oriented bar elements is almost as strong as motion between identically oriented elements does not necessarily contradict our conclusions. The optimal interstimulus interval probably would have been affected, had they taken identically oriented bar elements. Moreover, a serious problem with the measurement of the transition times in their experiment is the fact that matches between frames that are separated in time by a multiple of the stimulus interval time cannot be excluded. We therefore chose a relatively long frame time of $42 \mathrm{msec}$, to exclude motion paths between elements of frames that are not contiguous in time.

The effect of bar element orientation relative to motion direction was investigated for a considerable range of bar lengths and bar widths. It appears that distance ratio is a function of the length and width of the bar element. $R_{p s e}$ globally decreases as length increases, indicating a growing preference for motion in the direction of the element orientation. This preference declines at thicker bars. Local optima for the elongation of the bar elements exist. Significant changes in dominance of motion paths can be observed at bar length increments that can hardly be discriminated statically in bisection tasks, where $2 \%$ thresholds are observed (Levi, 1988; Volkmann, 1863). Furthermore, we have shown that an optimal width of the white center part exists for motion dominance between identical disk elements oriented along motion direction. When a modulation of receptive subfields is involved, characteristic lengths can be expected in the results. To reveal subfield fine structure, more experiments with structured image elements must be done.

In order to find a mechanistic explanation for the effects of element orientation relative to motion direction, we can postulate two aspects of motion detectors. These aspects may be distinguished conceptually by their order in the sequence of operations that a motion detector performs. First, some convolution of receptive subfields on the luminance distribution (and temporal filtering) takes place. Second, a directional selective detector must essentially perform a nonlinear operation that forms the output (also after temporal filtering). Third, to improve the signal-to-noise ratio, a spatial integration of the outputs of single motion detectors may take place. Both receptive field form, elongated in the tuning direction of the motion detector, and spatial integration in the direction of motion (van Doorn \& Koenderink, 1984) may account for our data. Although both operations are separated by a nonlinear operation (e.g., correlation), we have not yet been able to separate these two aspects in our experiment.

We have discussed our findings in geometrical terms such as element orientation, size, proximity, and receptive field forms. An alternative approach to analyzing visual motion stimuli is Fourier analysis, which has attracted many investigators (Adelson \& Bergen, 1985; Heeger, 1987; Watson \& Ahumada, 1985). In the Appendix, we show that differences in the low spatial frequency content of the two orthogonal motion paths in our stimuli can only account for the data if we assume that the receptive subfields of a motion detector are elongated in the motion direction. Moreover, we show that a Fourier perspective is inconsistent with the measured proximity relations. In the Appendix, such a Fourier analysis of our stimuli is given and discussed.

The dominance of motion along the element orientation is perhaps surprising with regard to the "aperture problem" (Adelson \& Movshon, 1982). The linear form of the bar elements in the pattern could set up constraints in velocity space. The component of velocity normal to the element orientation is well defined. In contrast, the velocity component along the element orientation is at best ambiguous. Consequently, this would predict a preference for motion orthogonal to the element orientation, which is inconsistent with our results.

A major question that remains is why an orientation of bar elements in the direction of motion is preferred in human motion vision. Suppose that the receptive fields of the motion detection system are indeed elongated in the 
direction of motion. For moving localized features (e.g., dots) and moving noise patterns, the elongation of receptive fields in the motion direction enables a precise discrimination of the direction of motion. However, discrimination of the speed is less precise. Consequently, this suggests that direction measurements are more important for visual tasks than measurements of speed are.

We conclude that it is not the orientation difference between bar elements but the orientation with respect to motion direction that is relevant to apparent motion.

\section{REFERENCES}

Adelson, E. H., \& Bergen, J. R. (1985). Spatio-temporal energy models for the perception of motion. Journal of the Optical Society of America A, 2, 284-299.

Adelson, E. H., Movson, J. A. (1982). Phenomenal coherence of moving visual patterns. Nature, 300, 523-525.

BRADDICK, O. J. (1974). A short range process in apparent motion. Vision Research, 14, 519-527.

Burr, D. C., Ross, J., \& Morrone, M. C. (1986). Smooth and sampled motion. Vision Research, 26, 643-652.

BURT, P., \& SPERLING, G. (1981). Time, distance and feature tradeoffs in visual apparent motion. Psychological Review, 88, 171-195.

Chang, J. J., \& Julesz, B. (1983). Displacement limits for spatial frequency filtered random dot cinematograms in apparent motion. Vision Research, 23, 1379-1385.

Chen, L. (1985). Topological structure in the perception of apparent motion. Perception, 14, 197-208.

CLEARY, R. (1989). Motion capture versus high spatial frequency masking. Investigative Ophthalmology \& Visual Science, 30(Suppl.), 388.

DUDA, R. O., \& HART, P. E. (1973). Pattern classification and scene analysis. New York: Wiley-Interscience.

FERNBERGER, S. W. (1934). New phenomena of apparent visual movement. American Journal of Psychology, 46, 309-314.

FrisBY, J. P. (1972). The effect of stimulus orientation on the phi phenomenon. Vision Research, 12, 1145-1166.

Gorea, A., \& Papathomas, V. (1988). A basic symmetry in motion perception. Perception, 17, A10. (From abstracts of the Proceedings of the 11th European Conference on Visual Perception)

GrEeN, M. (1986). What determines correspondence strength in apparent motion? Vision Research, 26, 599-607.

GreEN, M., \& OdoM, J. V. (1986). Correspondence matching in apparent motion: Evidence for three-dimensional spatial representation. Science, 233, 1427-1429.

HeEger, D. J. (1987). Optical flow from spatio-temporal filters. In Proceedings of the IEEE First International Conference on Computer Vision (pp. 181-190). London: IEEE Computer Society Press.

Koenderink, J. J., \& VAN DOORN, A. J. (1987). Representation of local geometry in the visual system. Biological Cybernetics, 55, 367-375.

Kolers, P. A. (1972). Aspects of motion perception. Oxford, England: Pergamon Press.

Kolers, P. A., \& Pomerantz, J. R. (1971). Figural change in apparent motion. Journal of Experimental Psychology, 87, 99-108.

KORTE, A. (1915). Kinematoscopische Untersuchungen. Zeitschrift fur Psychologie, 72, 193-206.

LEVI, D. M. (1988). "Weber's law" for position: Unconfounding the role of separation and eccentricity. Vision Research, 28, 597-603.

MarR, D. (1982). Vision. New York: Freeman.

MCKeE, S., \& Nakayama, K. (1984). The detection of motion in the peripheral visual field. Vision Research, 24, 25-32.

NAvoN, D. (1976). Irrelevance of figural identity for resolving ambiguities in apparent motion. Journal of Experimental Psychology: Human Perception \& Performance, 2, 130-138.

Orlansky, J. (1940). The effect of similarity and difference of form on apparent visual motion. Archives of Psychology, 246, 1-88.

Pantle, A., \& Picciano, L. A. (1976). A multistable movement dis- play: Evidence for two separate motion systems in human vision. Science, 193, 500-502.

RamachandRan, V. S., \& Anstis, S. M. (1986, June). The perception of apparent motion. Scientific American, pp. 80-87.

Ramachandran, V. S., \& Inada, V. (1985). Spatial and temporal characteristics of motion caption in random-dot patterns. Spatial Vision, 1, 55-67.

REICHARDT, W. (1969). Movement perception in insects. In W. Reichardt (Ed.), Processing of optical data by organisms and machines (pp. 465493). New York: Academic Press.

Shechter, S., Hochstein, S., \& Hillman, P. (1988). Shape similarity and distance disparity as apparent motion correspondence cues. Vision Research, 28, 1013-1021.

Shechter, S., Hochstein, S., \& Hillman, P. (1989). Size, flux and luminance effects in the apparent motion correspondence process. Vision Research, 29, 579-591.

Sperling, G., van Santen, J. P. H., \& Burt, P. J. (1985). Three theories of stroboscopic motion detection. Spatial Vision, 1, 47-56.

UlLMAN, S. (1978). Two-dimensionality of the correspondence process in apparent motion. Perception, 7, 683-693.

UlLmaN, S. (1980). The effect of similarity between bar segments on the correspondence strength in apparent motion. Perception, 9, 617-626.

VAN Doorn, A. J., \& Koenderink, J. J. (1984). Spatiotemporal integration in the detection of coherent motion. Vision Research, 24, 47-53.

van Santen, J. P. H., \& SPerling, G. (1985). Elaborated Reichardt detectors. Journal of the Optical Society of America A, 23, 300-321.

Volkmann, A. W. (1863). Physiologische Untersuchungen im Gebiete der Optik. Leipzig: Breitkopf und Hortel.

WATSON, A. B. (1986). Apparent motion occurs only between similar spatial frequencies. Vision Research, 26, 1727-1730.

Watson, A. B., \& Ahumada, A. J. (1985). Model of human visualmotion sensing. Journal of the Optical Society of America A, 2 , 322-342.

WatT, R. J., \& ANDrews, D. P. (1981). APE: Adaptive probit estimation of psychometric functions. Current Psychological Reviews, 1, 205-214.

WERTHEIMER, M. (1912). Experimentelle Studien über das Sehen von Bewegung. Zeitschrift für Psychologie, 61, 161-265.

Williams, D., \& SekUleR, R. (1984). Coherent global motion percepts from stochastic local motions. Vision Research, 24, 55-62.

\section{APPENDIX \\ A Fourier Analysis of the Stimulus}

We have discussed our findings in geometrical terms such as proximity, distance, size, and orientation. However, a discussion in "spectral" or "Fourier" terms could provide additional insights. One can think of two aspects concerning the spatial frequency contents of our stimuli that might account for our results: (1) the differences in the low spatial frequencies (the first harmonic that carries the motion) of the stimulus patterns in vertical and horizontal direction; and (2) the differences in the higher spatial frequencies, which can introduce ambiguous or reversed motion perception.

\section{First Harmonic}

The spatial frequency spectrum of the stimuli used is anisotropic; that is, it contains more energy for the first harmonic along the spatial axis colinear to the orientation of the elements than it does along the spatial axis orthogonal to the orientation of the elements, which could favor motion perception along the element orientation.

For example, let us take a pattern with elements oriented horizontally. The energy of the lowest spatial frequency com- 
ponent (first harmonic) in the horizontal direction is higher than the energy in the vertical direction. How can this energy difference explain the dominance of the horizontal motion path?

We can model the motion detection system with a bilocal correlator (Sperling et al., 1985) of receptive fields that sense the pattern. A receptive field can sense Fourier components in all directions, depending on its shape. Circular symmetric receptive fields sense the Fourier components in a horizontal direction as well as in a vertical direction. Thus, correlators with circular receptive fields cannot explain the dominance of the horizontal motion path. To explain the dominance of the horizontal path along the element orientation, the receptive fields of the motion detector (tuned to motion along the element orientation) must be more sensitive to low spatial frequencies along the element orientation than to low spatial frequencies orthogonal to the element orientation. This implies that the receptive fields of a motion detector are asymmetrical; that is, they are elongated in the tuning direction of the detector.

Consequently, reasoning in terms of element orientation or in terms of spatial frequency contents leads to the same conclusions.

\section{Higher Harmonics}

It can be suggested that because of differences in the frequency content for horizontal and vertical motion paths, the path orthogonal to element orientation contains more energy for the Fourier components that contribute to the reversed motion perception and less energy for the Fourier components that contribute to the motion direction than does the path that is colinear with element orientation. To give this thought a chance, we will analyze a motion path with oriented disk elements (diameter $D$, center bar width $W$, displacement $d_{v}$, and interelement distance $4 d_{v}$ ), oriented perpendicularly to motion direction. The Fourier spectrum in the direction of the displacement $d_{v}$ along an axis through the centers of the elements is discrete and contains frequencies $F_{n}=n / 4 d_{v}$ with energy $\left(A_{n}\right)^{2}$, where $A_{n}$ is:

$$
A_{n}=\frac{1}{\left(2 \pi d_{v} F_{n}\right)}\left[2 \sin \left(\pi F_{n} W\right)-\sin \left(\pi F_{n} D\right)\right] .
$$

The Fourier spectrum is actually the spectrum of an individual element, sampled at regular distances $\left(4 d_{v}\right)^{-1}$, due to the periodicity of the pattern. The Fourier components $F_{n}$ are displaced over a distance of $n / 4$ times their wavelength. Thus, in isolation, one would expect that components $F_{4 m}$ are static, that components $F_{4 m+1}$ contribute in the direction of the displacement, that components $F_{4 m+2}$ are ambiguous in the motion direction, and that components $F_{4 m+3}$ contribute in the reversed direction $(m=0,1,2,3, \ldots)$.

For example, in Figure 11, the energy of the components $F_{n}$ in the motion direction for a vertical path with horizontally oriented disk elements is plotted as a function of $\boldsymbol{n}$ for three vertical displacements $\left(d_{v}=10.4^{\prime}, 15.7^{\prime}\right.$, and 20.9'). All parameters are taken from the fourth experiment: observer P.W., diameter $D=18.6^{\prime}$, center width $W=8.7^{\prime}$.

Obviously, assumptions have to be made as to how these components are weighted in their contribution to the motion perception. There is some evidence that in discrete spectra the first harmonic $\left(F_{1}\right)$ dominates motion perception (Cleary, 1989;

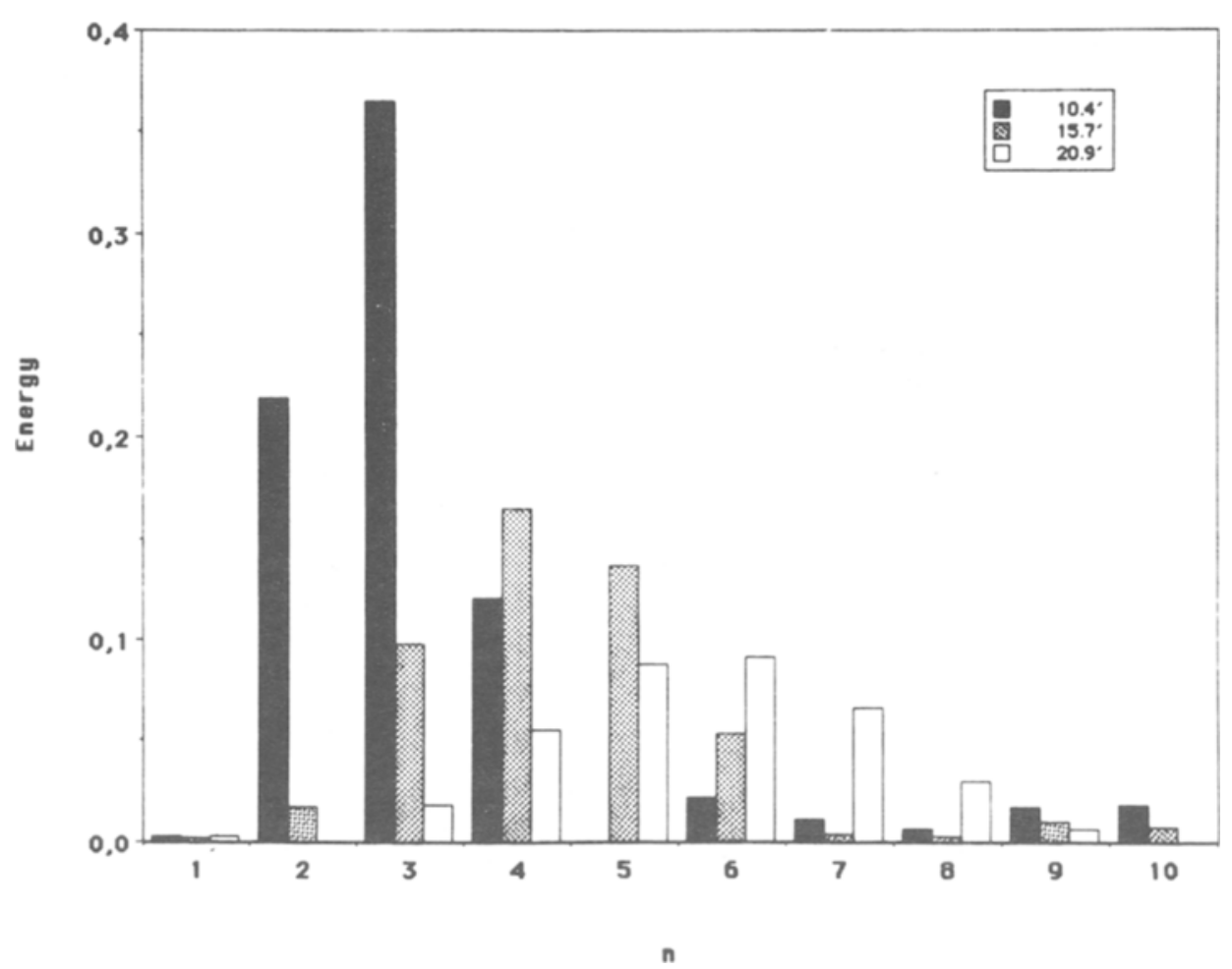

Figure 11. The energy $\left(A_{n}^{2}\right)$ of Fourier components $F_{n}(n=1, \ldots 10)$ along the motion path is plotted in arbitrary units for oriented disk elements $\left(W=8.7^{\prime}, D=18.6^{\prime}\right)$ oriented orthogonal to the motion path, for three displacements: $d_{v}=10.4^{\prime}$ (black bar), $d_{v}=15.7^{\prime}$ (dashed bar), and $d_{v}=20.9^{\prime}$ (white bar). 
Ramachandran \& Inada, 1985). Moreover, there are computational arguments to prefer a spatial band filter for the subunits with a tuning frequency $F_{t}=1 / 4 d_{v}=F_{1}$ (van Santen \& Sperling, 1985). Therefore, we expect only the low frequencies to be relevant to motion perception: $F_{1}$ (contributing in the direction of the displacement), $F_{2}$ (ambiguous), and $F_{3}$ (contributing in the reversed direction). It does not seem that the observed increase of dominance of the vertical motion path with decreasing vertical displacement $d_{v}$ in our experiments can be understood from the energy spectrum in Figure 11. The contribution of the reversed motion component $(n=3)$ increases strongly with decreasing displacement $d_{v}$ while the contribution of $F_{1}$ remains very small. This would predict a decrease of dominance in the direction of displacement $d_{v}$ as $d_{v}$ decreases, which is inconsis- tent with our findings. In fact, observer P.W. reported the opposite-a clear vertical motion perception for $d_{v}=10.4^{\prime}$ and horizontal motion perception for $d_{v}=20.9^{\prime}$. Moreover, the reversed motion arguments contradict the well-established monotonic proximity relations in general (Burt \& Sperling, 1981; Korte, 1915).

It should be noted that the periodicity of the pattern is not essential for our argumentation, since the spectrum of a periodic arrangement of elements is merely a sampled version of the continuous spectrum of an individual element. In conclusion, the above "spectral" arguments cannot explain our findings.

(Manuscript received July 24, 1989;

revision accepted for publication December 13, 1989.) 